ARTICLE

DOI: $10.1038 / \mathrm{s} 41467-017-02650-6$

\title{
Tbx5a lineage tracing shows cardiomyocyte plasticity during zebrafish heart regeneration
}

\author{
Héctor Sánchez-Iranzoํ, María Galardi-Castilla1, Carolina Minguillón²,7, Andrés Sanz-Morejón'1,3, \\ Juan Manuel González-Rosa ${ }^{1,4}$, Anastasia Felker ${ }^{5}$, Alexander Ernst ${ }^{3}$, Gabriela Guzmán-Martínez ${ }^{6}$, \\ Christian Mosimann (1D ${ }^{5}$ \& Nadia Mercader (iD ${ }^{1,3}$
}

During development, mesodermal progenitors from the first heart field (FHF) form a primitive cardiac tube, to which progenitors from the second heart field (SHF) are added. The contribution of FHF and SHF progenitors to the adult zebrafish heart has not been studied to date. Here we find, using genetic $t b \times 5 a$ lineage tracing tools, that the ventricular myocardium in the adult zebrafish is mainly derived from $t b \times 5 a^{+}$cells, with a small contribution from $t b \times 5 a$ - SHF progenitors. Notably, ablation of ventricular $t b \times 5 a^{+}$-derived cardiomyocytes in the embryo is compensated by expansion of SHF-derived cells. In the adult, tbx5a expression is restricted to the trabeculae and excluded from the outer cortical layer. tbx $5 a$-lineage tracing revealed that trabecular cardiomyocytes can switch their fate and differentiate into cortical myocardium during adult heart regeneration. We conclude that a high degree of cardiomyocyte cell fate plasticity contributes to efficient regeneration.

\footnotetext{
${ }^{1}$ Development of the Epicardium and Its Role during Regeneration Group, Centro Nacional de Investigaciones Cardiovasculares (CNIC-ISCIII), Melchor Fernández Almagro 3, 28029 Madrid, Spain. ${ }^{2}$ CSIC-Institut de Biologia Molecular de Barcelona Parc Científic de Barcelona C/ Baldiri i Reixac, 1008028 Barcelona, Spain. ${ }^{3}$ Institute of Anatomy, University of Bern, 3000 Bern 9, Switzerland. ${ }^{4}$ Cardiovascular Research Center, Massachusetts General Hospital and Harvard Medical School, Boston, MA 02114, USA. ${ }^{5}$ Institute of Molecular Life Sciences, University of Zürich, 8057 Zürich, Switzerland. ${ }^{6}$ Hospital Universitario La Paz, IdiPAZ, 28046 Madrid, Spain. ${ }^{7}$ Present address: Barcelonabeta Brain Research Center, Pasqual Maragall Foundation, 08005 Barcelona, Spain. Correspondence and requests for materials should be addressed to N.M. (email: nadia.mercader@ana.unibe.ch)
} 
T he zebrafish is an established model organism to study heart regeneration, in which pre-existing cardiomyocytes proliferate to replace the lost myocardium ${ }^{1-3}$. The zebrafish myocardium is formed by an inner trabecular layer, a thin primordial layer, and an outer cortical layer. During embryonic development, cells from the primordial layer give rise to trabeculae $^{4}$. Later, in the juvenile, trabecular cardiomyocytes breach the primordial layer and form the cortical myocardium ${ }^{5,6}$. Previous clonal analysis suggested that during adult heart regeneration, the cortical myocardium is rebuilt by cardiomyocytes from the same layer ${ }^{5}$, suggesting some degree of commitment to a particular myocardial layer. A higher degree of plasticity had been observed in the embryonic heart, whereby atrial cardiomyocytes were reported to be able to regenerate the cardiac ventricle ${ }^{7}$.

The vertebrate heart is formed from mesodermal precursor cells derived from the first and second heart fields, also called FHF and SHF, respectively. Cells from the FHF form the embryonic heart tube to which cells from the SHF are added to allow further growth. FHF and SHF progenitors have also been described in the zebrafish ${ }^{8-12}$. Regulatory elements from the draculin $(\mathrm{drl})$ gene have been used to label a population of lateral plate mesoderm progenitors that give rise to cardiomyocytes of the primitive heart tube, providing a genetic marker to trace FHFderived myocardium ${ }^{11}$. Latent tgf beta binding protein 3 (ltbp3) expression marks a distal $d r l$-negative domain and has been proposed as a SHF marker ${ }^{12}$. Of note, neither $d r l$ nor $l t b p 3$ are established FHF and SHF markers in other vertebrates including mammals ${ }^{11}$.

In the mouse, retrospective clonal analysis revealed that FHFderived cells predominantly give rise to the left ventricle, whereas the SHF yields the right ventricle and large parts of the atria ${ }^{13,14}$. Further studies on the contribution of cardiac precursor cells to the mammalian heart come from genetic lineage tracing approaches, which are based on following the fate of cells that expressed a particular gene at a given time point of embryonic development ${ }^{15-17}$. A key gene used to trace the fate of cardiac precursor cells is Tbx5, encoding a T-box transcription factor, which serves as an early cardiac marker expressed in the developing heart tube ${ }^{18,19}$. Genetic lineage tracing analysis of Tbx5expressing cells has revealed a strong contribution to the left ventricular myocardium, recapitulating the fate of FHF progenitors in this cardiac chamber ${ }^{20}$

Zebrafish have two Tbx5-encoding genes, $t b \times 5 a$ and $t b x 5 b$; $t b x 5 a$ expression levels during heart development are higher than those of $t b x 5 b$, and $t b \times 5 a$ mutants recapitulate key phenotypes of mammalian $T b x 5$ perturbations ${ }^{21-23}$. We thus decided to generate genetic tools to study the contribution of the FHF to the adult ventricle during homeostasis and regeneration in the zebrafish using regulatory sequences of $t b x 5 a$. We describe that in zebrafish the early heart tube is formed by $t b x 5 a$-derived cardiomyocytes to which SHF-derived $t b \times 5 a^{-}$cardiomyocytes are added. Even though their contribution to the adult heart is minimal, SHF-derived cells can give rise to a fully functional ventricle if $t b x 5 a$-derived cells are genetically ablated. In the adult heart, $t b x 5 a$ expression is persistent only in the trabecular myocardium, allowing to distinguish this layer from the cortical myocardium. Genetic fate mapping of trabecular $t b \times 5 a^{+}$cells shows that these cells contribute to regenerate the outer cortical layer. Together, our findings show a high degree of cardiomyocyte fate plasticity during zebrafish heart regeneration, during both embryonic stages and in the adult.

\section{Results}

Contribution of $t b x 5 a^{+}$cardiomyocytes to the adult ventricle. We used the fluorescent bacterial artificial chromosome (BAC)

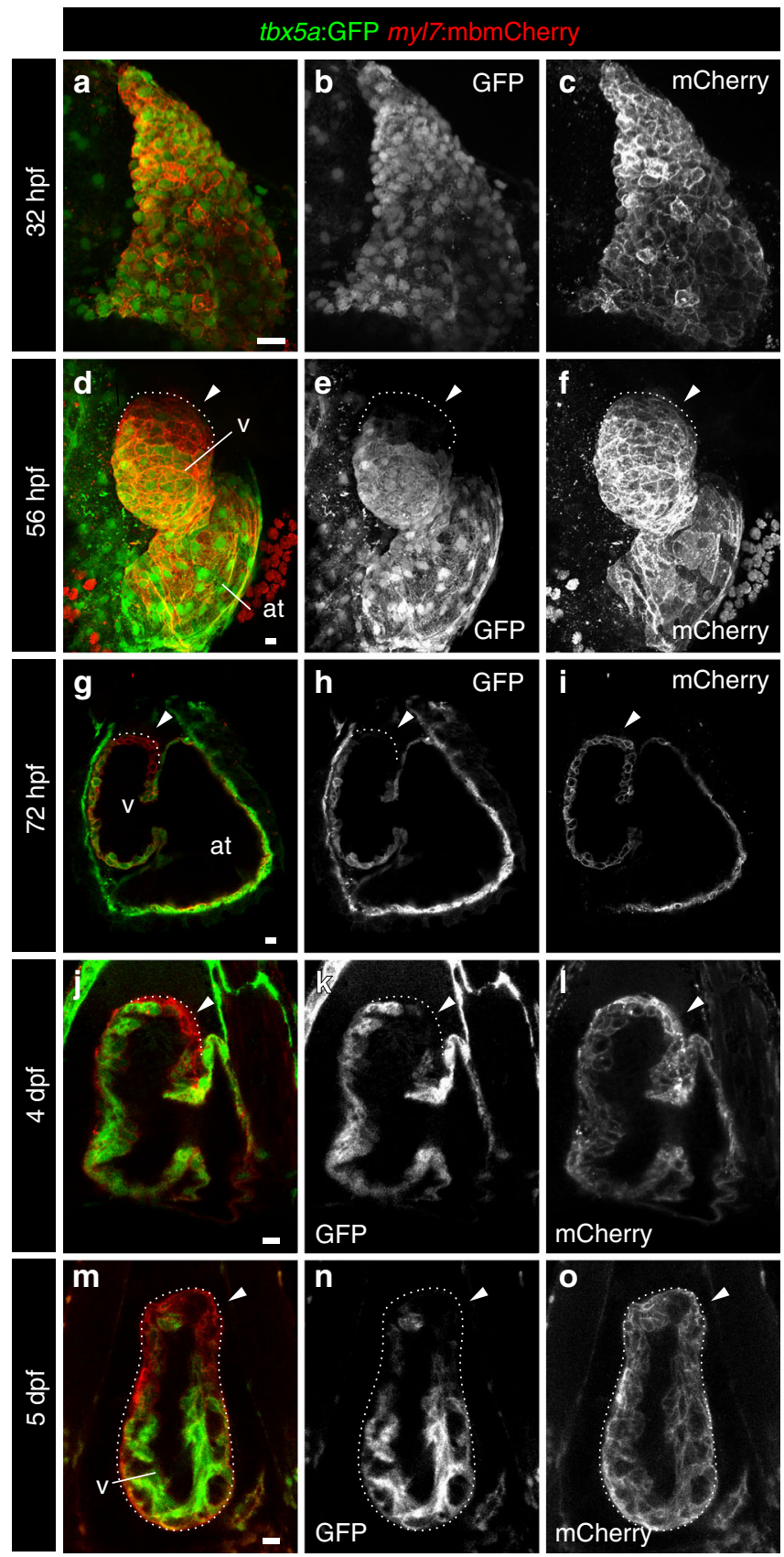

Fig. 1 Expression profile of tbx5a-positive cardiomyocytes in embryonic zebrafish hearts. a-f Whole-mount immunofluorescence of tbx5a:GFP;my/7:

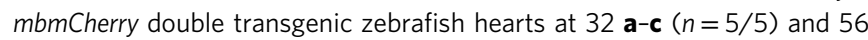
hours postfertilisation (hpf) d-f $(n=3 / 3)$. g-I Confocal optical sections of tbx5a:GFP;myl7:mbmCherry hearts at $72 \mathrm{hpf} \mathbf{g}-\mathbf{i}(n=5 / 5), 4$ days postfertilisation (dpf) j-I $(n=9 / 9)$, and $5 \mathrm{dpf}(\mathbf{m}-\mathbf{0} ; n=6 / 6)$. GFP (green) labels $t b \times 5 a^{+}$cells and mCherry (red) marks cells expressing the panmyocardial marker myosin light chain 7 (myl7). Shown are ventral views, cranial is to the top. At $32 \mathrm{hpf}$ all cardiomyocytes are tbx5a:GFP+ but at 56 $72 \mathrm{hpf}, 4$, and $5 \mathrm{dpf}$ tbx5a:GFP- cardiomyocytes can be observed in the distal ventricle (arrowheads). The atrioventricular canal and large portions of the atrium are also $\mathrm{GFP}^{+}$. at, atrium; v, ventricle; Scale bars, $10 \mu \mathrm{m}$

transgenic reporter line $t b x 5 a: G F P$, driving green fluorescent protein (GFP) expression under the control of the tbx5a cisregulatory elements ${ }^{24}$. The overall GFP expression pattern of tbx5a:GFP recapitulated that of endogenous $t b \times 5 a$ expression in embryos (Supplementary Fig. 1a, b; $n=7 / 7$ ). We analysed the 


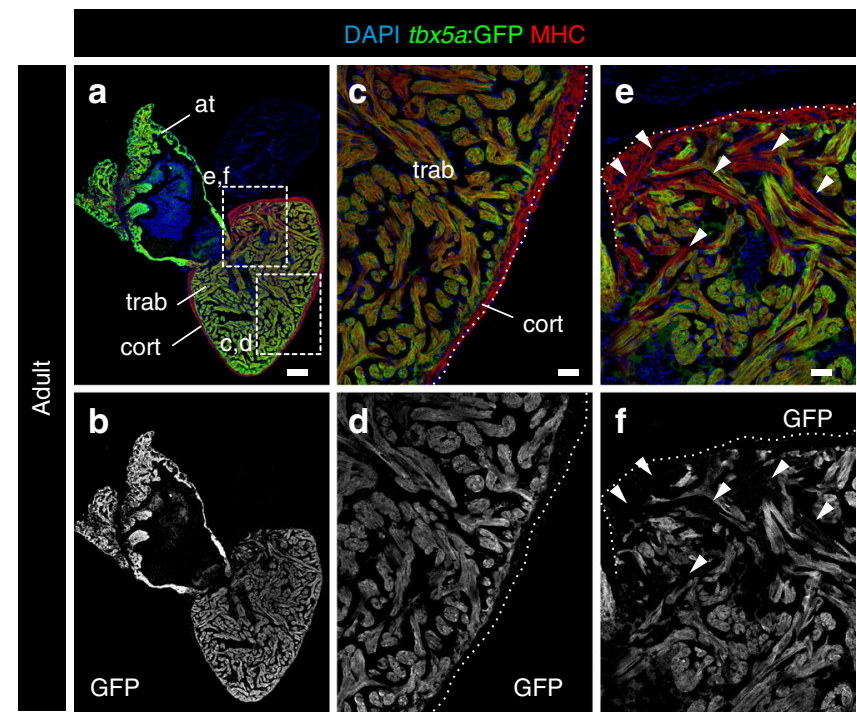

Fig. 2 Expression profile of tbx5a-positive cardiomyocytes in adult zebrafish hearts. a, b Sagittal sections through tbx5a:GFP adult uninjured heart immunostained with GFP (green) and Myosin Heavy Chain (MHC; red). Nuclei are counterstained with DAPI (blue). c-e Zoomed views of boxed area in $\mathbf{a} . \mathbf{b}, \mathbf{d}$, f Single channels for GFP. The trabecular myocardium is tbx $5 a: G F^{+}$, whereas the cortical layer is $t b \times 5 a: G^{-} P^{-}$. Note $t b \times 5 a: G F P^{-}$ cardiomyocytes (arrowheads) in the basal part of the ventricle close to the atrioventricular canal $(n=13 / 13)$. at, atrium; cort, cortical layer; trab, trabecular layer. Scale bars, a, b $100 \mu \mathrm{m}$ and c-f $25 \mu \mathrm{m}$

expression of $t b x 5 a: G F P$ in the early heart tube, before the addition of SHF progenitors. At this stage, the heart tube is formed by a single sheet of cardiomyocytes named the primordial layer ${ }^{5}$. Homogeneous $t b x 5 a$ :GFP expression was detected in the whole embryonic myocardium at 32 hours postfertilisation (hpf) (Fig. 1a-c; $n=5 / 5$ ). After this stage, at $56 \mathrm{hpf}$, (Fig. 1d-f; $n=3 / 3$ ), a $\mathrm{GFP}^{-}$region of myocardium started to become visible in the cranial portion of the heart tube, which remained reporternegative at $72 \mathrm{hpf}$ (Fig. 1g-i; Supplementary Movie 1, $n=5 / 5$ ), 4 days postfertilisation (dpf) (Fig. $1 \mathrm{j}-1 ; n=9 / 9$ ), and at $5 \mathrm{dpf}$ (Fig. $1 \mathrm{~m}-0 ; n=6 / 6$ ), concomitant with the reported addition of SHF progenitors to the ventricular myocardium during these stages ${ }^{8,12}$. The GFP expression pattern was confirmed by $g f p$ messenger RNA in situ hybridisation on heart sections of embryos at 2-5 dpf (Supplementary Fig. 1c-g). Comparison of the expression domains at 56 and $72 \mathrm{hpf}$ between $t b x 5 a$ :GFP and the previously reported FHF marker $d r l$ mCherry revealed an overlap of both domains in the proximal part of the ventricle close to the atrioventricular canal (AVC) and in the outer curvature of the ventricle (Supplementary Fig. 2a-h and Supplementary Movies 2 and 3; $56 \mathrm{hpf}, n=5 / 5 ; 72 \mathrm{hpf}, n=7 / 7$ ). Of note, the expression domain of $t b \times 5 a$ :GFP was more extensive toward the distal myocardium. This might indicate that $d r l$ expression is downregulated faster in FHF-derived cells than $t b x 5 a$, or that part of the proximal SHF-derived myocardium expresses $t b x 5 a$. Nonetheless, a clear tbx5a:GFP/drl:mCherry double-negative domain was present in the inner curvature and distal-most portion of the larval ventricle (Supplementary Fig. 2a-h and Supplementary Movies 2 and 3). This $t b x 5 a: \mathrm{GFP}^{-}$region was further expressing transiently injected ltbp3:TagRFP-2A-Cre ${ }^{12}$, indicating SHF-derived ventricular myocardium (Supplementary Fig. 2i-1 and Supplementary Movie 4). Thus, in the embryonic heart, tbx5a expression marks cardiomyocytes in the primitive heart tube and is absent from a population of distally located ventricular cardiomyocytes, which from their expression pattern and temporal appearance match with previous descriptions of the most distal SHF-derived cells ${ }^{12}$.

We next sought to chart $t b x 5 a$ expression in the adult zebrafish heart. During zebrafish heart maturation, a third myocardial layer forms enveloping the primordial layer, named cortical layer. Clonal analysis suggested that these myocardial layer derives from trabecular cardiomyocytes ${ }^{5}$. We still detected high levels of endogenous $t b x 5 a$ mRNA and tbx5a:GFP expression in the adult zebrafish ventricle (Fig. 2a, b and Supplementary Fig. 3; $n=8 / 8$ ). $t b \times 5 a$ :GFP expression was restricted to the trabecular myocardium and absent from the cortical layer (Fig. 2c, d), coinciding with the recently reported pattern obtained using an enhancer fragment located $16 \mathrm{~kb}$ upstream of the $t b \times 5 a$ transcription start site $^{25}$. Expression spread through most of the trabecular myocardium, with a gradient from the apex (high expression) to the basal part close to the bulbus arteriosus (BA) (lower expression levels). In addition, we observed a region in the basal ventricle, close to the atrium, in which we detected $\mathrm{GFP}^{-}$ trabecular cardiomyocytes. No GFP expression can be detected in these cells beyond background and we therefore refer to this myocardial territory as basal $t b x 5 a^{-}$region (Fig. $2 \mathrm{e}, \mathrm{f} ; n=13 / 13$; Supplementary Fig. 4).

$t b \times 5 a^{-}$cardiomyocytes in the adult heart could derive from embryonic $t b \times 5 a^{+}$cells that switched off their expression or from progenitor cells that never expressed tbx5a. To test these possibilities, we generated transgenic lines for inducible genetic fate mapping of $t b x 5 a$-expressing cells (Fig. 3a). In doubletransgenic tbx5a:mCherry-p2A-CreER ${ }^{T 2} ;$ ubb:loxP-lacZ-STOPloxP-GFP ${ }^{26}$ embryos, mCherry labels $t b x 5 a$-expressing cells and GFP labels the progeny of $t b x 5 a^{+}$cells that expressed CreER ${ }^{\mathrm{T} 2}$ recombinase at the time of 4-Hydroxytamoxifen (4-OHT)induced recombination. This transgenic combination was not leaky and GFP-expressing cells were visible only upon 4-OHT administration (Fig. 3b; $n=5 / 5$ ). When recombined during embryogenesis, $\mathrm{GFP}^{+}$cells were found in the atrium and ventricle of the larval heart but never in the distal portion of the ventricular myocardium (Fig. 3c-f; 8/8; Supplementary Fig. 5 and Supplementary Movie 5, $n=9 / 9$ ). Few epicardial cells were also tbx5aderived, consistent with $t b \times 5 a$ :tdTomato expression observed in a subset of embryonic epicardial cells (Supplementary Fig. 6; $n=6$ / $7)$. When analysed in adult hearts, the cortical layer was mCherry - , in agreement with our observations using the reporter $t b x 5 a$ : GFP. Nonetheless, most of the cortical layer was $\mathrm{GFP}^{+}$and thus derived from a $t b \times 5 a^{+}$embryonic cell population that switched off $t b x 5 a$ expression (Fig. 3g-n; $n=5 / 5$; Supplementary Fig. 7, 8).

Akin to the GFP pattern observed in $t b x 5 a$ :GFP adult hearts, a basal domain of the ventricle close to the BA was also mCherry ${ }^{-}$ (Fig. $3 \mathrm{~g}-\mathrm{j}$ and $3 \mathrm{o}-\mathrm{r}$, and Supplementary Fig. 7, $8 ; n=5 / 5$ ). Furthermore, this domain was also $\mathrm{GFP}^{-}$, suggesting that it derives from the $t b \times 5 a^{-}$distal embryonic ventricle. Thus, we propose that $t b \times 5 a^{+}$-derived cells in the adult heart predominantly comprise the FHF derivatives of the embryonic heart and the $t b \times 5 a^{-}$domain in the basal ventricle close to the atrium and BA is SHF-derived. Taken together, our genetic lineage tracing of embryonic myocardial populations into adulthood in zebrafish reveal a fate compartmentalisation of $t b x 5 a^{-}$and $t b x 5 a^{+}$-derived cardiomyocytes starting in embryogenesis that results in distinguishable ventricle domains in the absence of septation.

SHF progenitors compensate ablated $t b x 5 a^{+}$cardiomyocytes. Previous results have indicated that atrial cardiomyocytes acquire a ventricular phenotype upon injury in the embryonic heart ${ }^{\top}$. To explore whether SHF progenitors can compensate for a loss of FHF-derived cells, we generated vmhcl:loxP-tagBFP-loxP$m$ Cherry-NTR, a transgenic line to specifically mark and ablate 


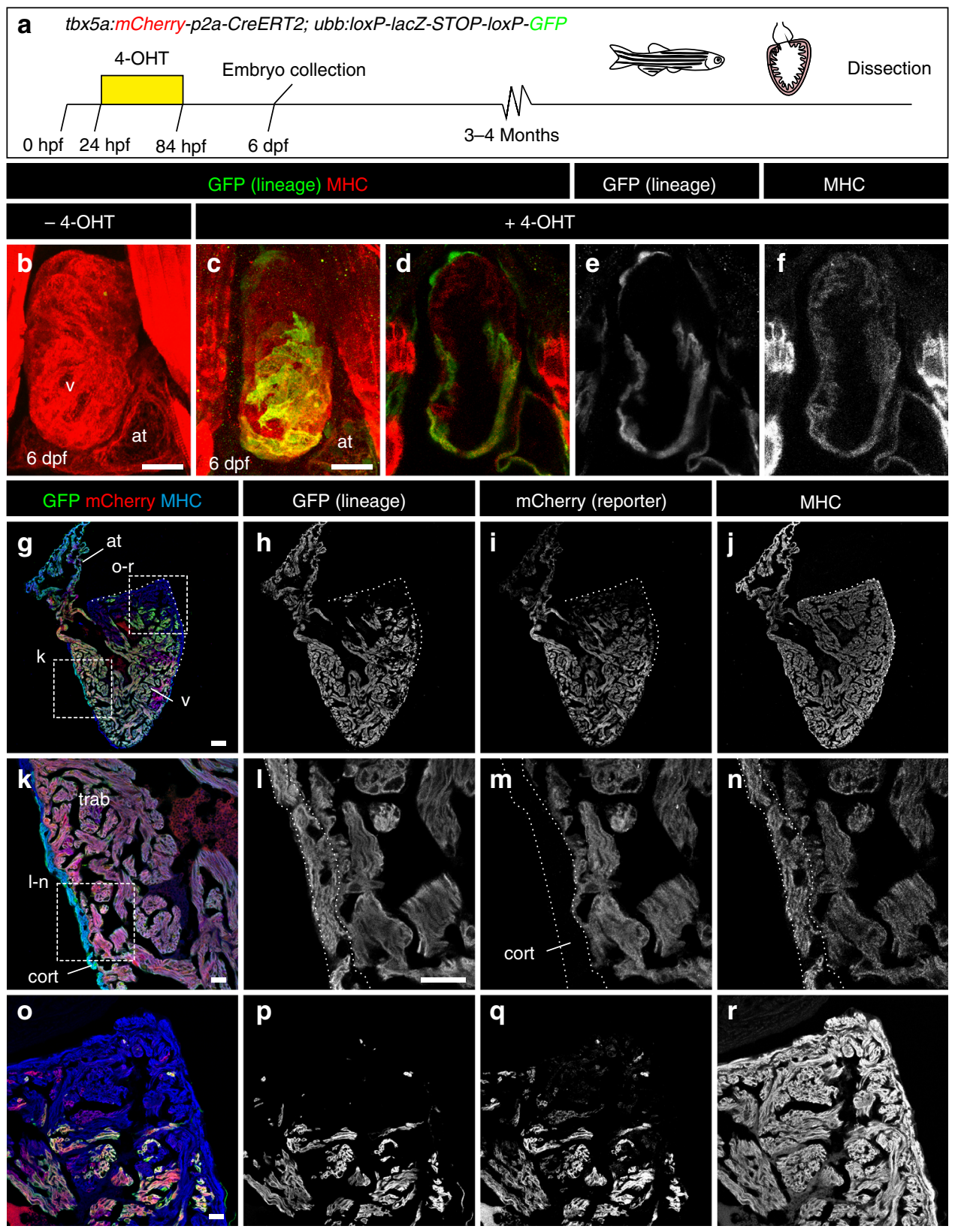

Fig. 3 Fate mapping of $t b \times 5 a$-derived cells during cardiac development. a tbx5a:mCherry-p2A-CreER ${ }^{T 2}$; ubb:loxP-LacZ-STOP-loxP-GFP hearts fixed at different developmental stages. mCherry marks tbx5a-expressing cells; GFP marks tbx5a-derived cells. b-f Whole-mount ventral view of hearts at 6 days postfertilisation (dpf) stained for GFP (green) and Myosin Heavy Chain (MHC, red). b In the absence of 4- Hydroxytamoxifen $(4-\mathrm{OHT})$ administration, no $\mathrm{GFP}^{+}$cells are visible $(n=5 / 5)$. c-f 4-OHT was added from 24 to 84 hours postfertilisation (hpf). GFP expression is observed in the proximal part of the ventricle; $n=8 / 8$. In some cases, GFP expression was also found in epicardial cells located in the distal part of the ventricle. $\mathbf{g}$-r Immunofluorescence staining of adult heart sections recombined as in c. Shown are merged and single channels for GFP (green), mCherry (red), and antiMHC staining (blue); $n=5 / 5$. at, atrium; cort, cortical layer; trab, trabecular layer; $v$, ventricle. Scale bars, $\mathbf{g} 100 \mu \mathrm{m}$ and $\mathbf{b}, \mathbf{c}, \mathbf{k}, \mathbf{I}, \mathbf{0} 25 \mu \mathrm{m}$

ventricular cardiomyocytes using the ventricular myosin heavy chain like ( $\mathrm{vmhcl}$ ) cis-regulatory elements (Fig. 4a; Supplementary Fig. 9; $n=10 / 10)$. vmhcl is expressed exclusively in ventricular and not atrial cardiomyocytes ${ }^{27}$. We crossed vmhcl:loxP-tagBFPloxP-mCherry-NTR with $t b x 5 a: C r e E R^{T 2}$. This allowed to lineagetrace $t b x 5 a^{+}\left(\right.$mCherry $\left.^{+}\right)$and $t b x 5 a^{-}$ventricular cardiomyocytes $\left(\operatorname{tagBFP}{ }^{+}\right)$. Furthermore, this genetic strategy allows the ablation of $t b x 5 a^{+}$ventricular cardiomyocytes by adding Metronidazole (Mtz) to induce cell death in nitroreductase (NTR)-expressing cells $^{28}$. Administration of 4-OHT from 24 to $48 \mathrm{hpf}$ induced the expression of mCherry-NTR in tbx5a-derived cardiomyocytes in the trabecular and primordial layer of the embryonic ventricle, with the exception of the distal-most part at the cranial pole (Fig. 4b, c, $n=7 / 7$ ). In adult hearts, most ventricular cardiomyocytes were mCherry ${ }^{+}$. Only a small portion of cardiomyocytes close to the BA and AVC was tagBFP ${ }^{+}$(Fig. 4d, e), coinciding with the results obtained doing lineage tracing using tbx5a:mCherry-p2A-CreER ${ }^{T 2}$; ubb:loxP-lacZ-STOP-loxP-GFP. To specifically ablate mCherry ${ }^{+}$cardiomyocytes we administered Mtz from 4 to $7 \mathrm{dpf}$ (Supplementary Fig. 10). Surprisingly, 30 days later, a complete ventricle had regenerated from tagBFP ${ }^{+}$ cardiomyocytes, suggesting that SHF-derived ventricular cardiomyocytes are able to compensate the genetic ablation of the FHFderived ventricular myocardium $(n=6 / 6 ;$ Fig. $4 \mathrm{f}-\mathrm{h}$ and 


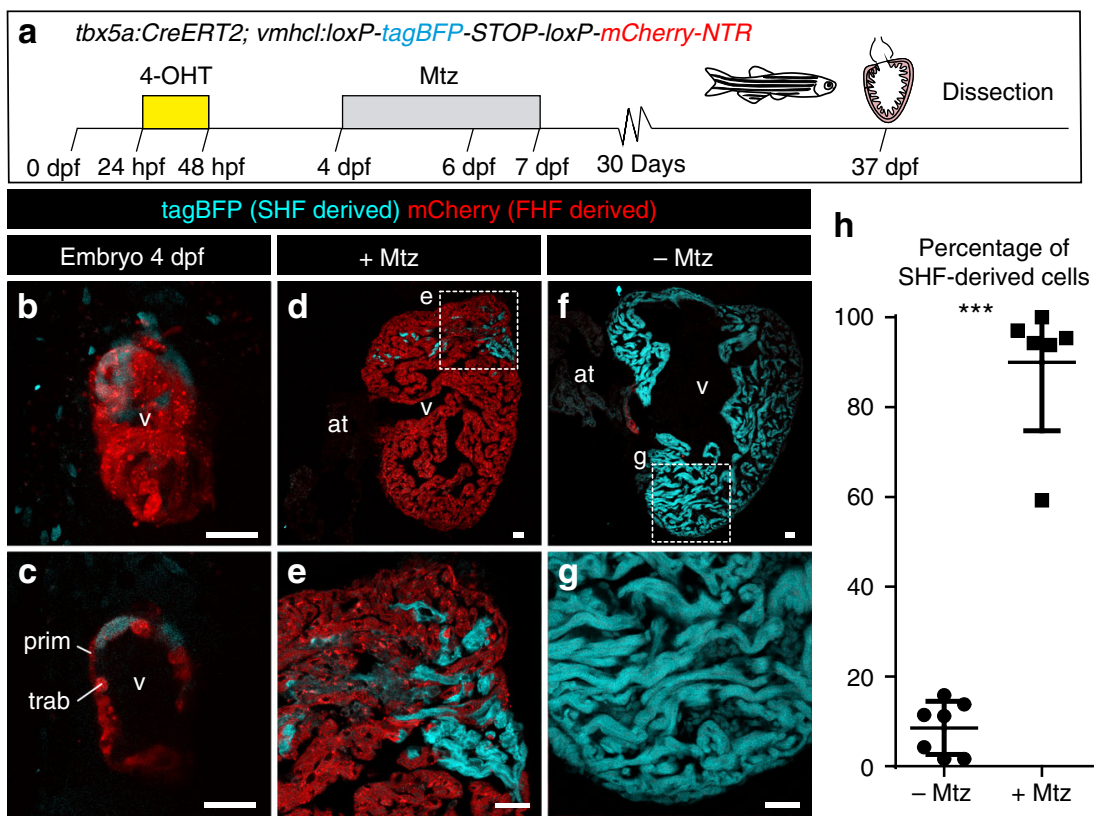

Fig. 4 Genetic ablation of $t b \times 5 a$-derived ventricular cardiomyocytes. a $t b \times 5 a^{+}$ventricular cardiomyocytes were genetically ablated in $t b \times 5 a: C r e E R^{T 2} ; v m h c l$ : loxP-tagBFP-loxP-mCherry-NTR double transgenic zebrafish. Recombination was induced by administration of 4-Hydroxytamoxifen (4-OHT). Cell ablation was induced by administration of Metronidazole (Mtz) from 4 to 7 days postfertilisation. $\mathbf{b}, \mathbf{c}$ Ventral views of larval hearts at $4 \mathrm{dpf}$ (maximal projection and optical section, respectively). Anterior is to the top. The proximal ventricle, including primordial layer and trabeculae, is completely $\mathrm{mCherry}{ }^{+}$and the distal ventricle is blue $(\operatorname{tagBFP})^{+} ; n=7 / 7$. d, e Sagittal section of the ventricle of an adult recombined heart. Most cells are $\mathrm{mCherry}^{+}$. Only the tbx5 $a^{-}$ region is tagBFP. $n=7 / 7 . \mathbf{f}, \mathbf{g}$, Sagittal section of a Mtz-treated fish. Most of the cardiomyocytes are tagBFP $; n=6 / 6$. $\mathbf{h}$, Quantification of the percentage of myocardium that is tagBFP ${ }^{+}$(SHF-derived), mean $\pm \mathrm{SD}$; ${ }^{* \star} P<0.0001$ by two-tailed unpaired $t$-test. at, atrium; prim, primordial layer; SHF, second heart field; trab, trabeculae; $v$, ventricle. Scale bars, $25 \mu \mathrm{m}$

Supplementary Fig. 11). In the experimental setup all atrial cardiomyocytes are $t b x 5 a$ lineage-derived and thus would become mCherry ${ }^{+}$if they contributed to the ventricle (Supplementary Fig. 5 and Supplementary Movie 5, $n=9 / 9$ ). Thus, our results reveal that ventricular $t b \times 5 a^{-}$SHF-derived cells compensated for the loss of $t b x 5 a^{+}$ventricular cardiomyocytes. To confirm ablation of $t b x 5 a$-derived mCherry-NTR expressing cells, we performed terminal deoxynucleotidyl transferase (TdT)-mediated dUTP nick end labeling (TUNEL) staining to detect apoptosis (Fig. 5a-g; $n=8$ larvae $+\mathrm{Mtz}$ group and $n=8-\mathrm{Mtz}$ group) and detected a significant increase of $\mathrm{TUNEL}^{+} \mathrm{mCherry}^{+}$trabecular and primordial cardiomyocytes in the Mtz-treated group. In agreement, tagBFP ${ }^{+}$ventricular cardiomyocytes incorporated significantly more 5-bBromo-2'-deoxyuridine (BrdU) upon $\mathrm{Mtz}$ treatment, indicating proliferation of this cell population (Fig. 5h-m).

Ablation of $t b x 5 a$-derived ventricular cardiomyocytes led to a transient impairment of cardiac function. At $7 \mathrm{dpf}$, right after the Mtz treatment, the ventricles revealed an irregular shape and displayed diminished pumping efficiency (Supplementary Fig. 12a-e and Supplementary Movie 6). However, mortality was not significantly increased (Supplementary Fig. 12f). Indeed, in the adults, the regenerated hearts revealed normal ventricular pumping efficiency (Supplementary Fig. 12g). Taken together, our results demonstrate that the loss of FHF-derived cardiomyocytes is compensated by an expansion of SHF-derived cardiomyocytes.

Cardiomyocyte plasticity during adult heart regeneration. We next explored whether this observed cardiomyocyte plasticity remains in the adult. Upon cryoinjury, cardiomyocytes at the site of injury proliferate and rebuild the lost cortical and trabecular myocardium $^{1,2}$. Using our transgenic tools, which genetically discriminate between cortical and trabecular cardiomyocytes, we investigated the plasticity of trabecular cardiomyocyte cell fate during regeneration. First, we analysed the expression of $t b x 5 a$ : GFP during heart regeneration using cryoinjury (Supplementary Fig. 13a). tbx5a:GFP was highly expressed in uninjured trabeculae at all stages analysed. At 1 days postinjury (dpi), no GFP expression could be found in the cortical layer (Supplementary Fig. 13b-f; $n=4 / 4$ ). In contrast, at 3 dpi (Supplementary Fig. 13g-k; $n=4 / 5$ ), 7 dpi (Supplementary Fig. 13l-p; $n=3 / 3$ ), and 21 dpi (Supplementary Fig. 13q-u; $n=4 / 4$ ), few $t b x 5 a: G_{F P}{ }^{+}$ cardiomyocytes could be observed in the cortical layer close to the injured area. Nonetheless, at $130 \mathrm{dpi}$, the regenerated cortical myocardium was tbx5a:GFP ${ }^{-}$(Supplementary Fig. $13 \mathrm{v}-\mathrm{z} ; n=4$ / 4). This transient expression of $t b x 5 a$ in cortical cardiomyocytes can have two explanations: (a) $t b x 5 a$ is re-expressed transiently in the cortical layer during regeneration, or (b) trabecular cardiomyocytes are contributing to the new cortical layer.

To evaluate these two possibilities, tbx5a-derived cells were genetically traced using tbx5a:mCherry-p2A-CreER ${ }^{T 2} ; u b b: l o x P$ $S T O P$-loxP-GFP double-transgenic fish (Fig. 6a). Upon recombination, GFP expression was restricted to the trabeculae in uninjured hearts (Fig. 6b-g; $n=7 / 7$ ) and we only rarely detected $\mathrm{GFP}^{+}$cells in the cortical myocardium (two cells in one out of six completely sectioned hearts, Supplementary Fig. 14a-f). By contrast, we observed $\mathrm{GFP}^{+} / \mathrm{mCherry}^{-}$cells in the regenerated cortical layer at $21 \mathrm{dpi}$ (Fig. 6h-m; $n=6 / 7$ ) and $90 \mathrm{dpi}$ (Fig. 6n, $o-s ; n=6 / 6)$, indicating that these cells derived from $t b x 5 a^{+}$cells had switched off $t b x 5 a$ expression. In the remote area, $\mathrm{GFP}^{+}$cells remained restricted to the trabecular layer even upon cryoinjury (Fig. 6t-x; $n=5 / 6$ ). Based on the percentage of trabecular cardiomyocytes that are $\mathrm{GFP}^{+}$, the recombination efficiency was estimated to be $10 \pm 6 \%$. In the newly formed cortical layer, $7 \pm$ 


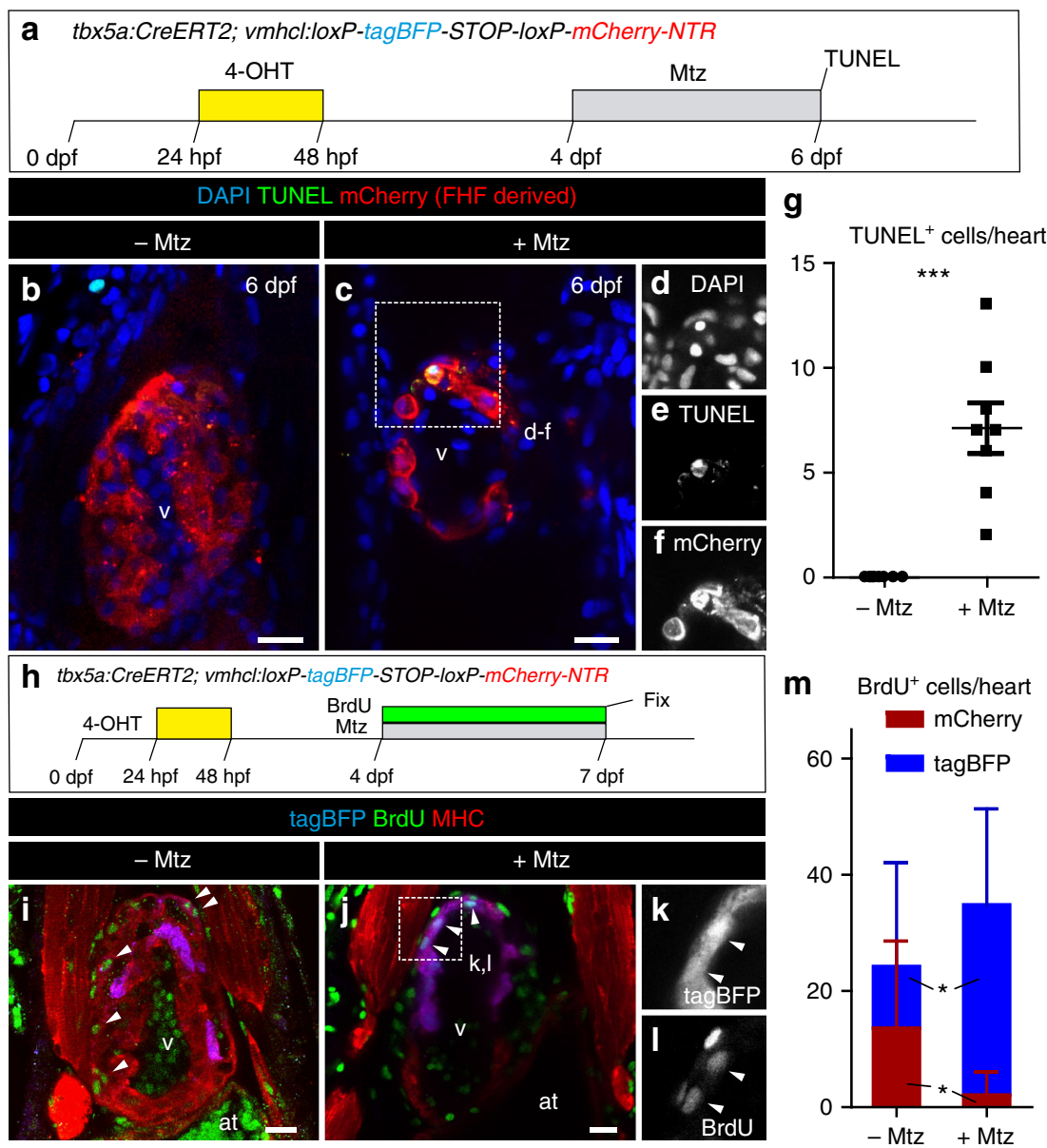

Fig. 5 Apoptosis and cell proliferation upon genetic ablation of tbx5a-derived ventricular cardiomyocytes. a tbx $5 a^{+}$ventricular cardiomyocytes were genetically ablated in tbx5a:CreER ${ }^{T 2}$;vmhcl:loxP-tagBFP-loxP-mCherry-NTR double transgenic zebrafish. Recombination was induced by administration of 4Hydroxytamoxifen (4-OHT). Cell ablation was induced by administration of Metronidazole (Mtz) from 4 to 7 days postfertilisation (dpf). b, c Optical sections of $6 \mathrm{dpf}$ fish that had been treated with 4-OHT and Mtz as indicated in $\mathbf{a}(\mathbf{b})$ or only with 4-OHT $\mathbf{c}$ immunostained for mCherry (red) and terminal deoxynucleotidyl transferase (TdT)-mediated dUTP nick end labeling (TUNEL) (green). Note that some rounded $\mathrm{mCherry}^{+}$cells are TUNEL ${ }^{+}$. d-f Single channels of selected area in $\mathbf{c} . \mathbf{g}$ Quantification of the number of TUNEL ${ }^{+}$trabecular and cortical $\mathrm{mCherry}^{+}$cardiomyocytes per heart from animals of the + Mtz $(n=8)$ and $-M t z(n=8)$ group, mean \pm SD; ${ }^{\star \star \star} P=0.0004$ by Mann-Whitney non-parametric $t$-test. h Schematic representation of the 5-Bromo-2 -deoxyuridine (BrdU) treatment to assess proliferation. $\mathbf{i}$, j Fish were treated with 4-OHT and BrdU i or with 4-OHT, Mtz, and BrdU j. $\mathbf{k}, \mathbf{I}$ Single channels of the boxed area in $\mathbf{j} . \mathbf{m}$ Quantification of $\mathrm{BrdU}^{+} / \mathrm{mCherry}^{+}$and $\mathrm{BrdU}^{+} / \operatorname{tagBFP}{ }^{+}$cells per heart. Shown are means $\pm \mathrm{SD}(n=8$ for $-\mathrm{Mtz}$ hearts and $n=11$ for + Mtz hearts, from two separate independent experiments) ${ }^{\star} P=0.0240$ for tagBFP ${ }^{+}$cells and $P=0.0371$ for mCherry ${ }^{+}$cells by two-tailed $t$-test. at, atrium; hpf, hours postfertilization; $v$, ventricle. Scale bars, $25 \mu \mathrm{m}$

$4 \%$ of cardiomyocytes are $\mathrm{GFP}^{+}(n=10)$. Therefore, trabeculae might contribute to a higher extent to the regenerating cortical layer than estimated by GFP signal detection.

To confirm that these cells came from $t b x 5 a^{+}$trabeculae and were not produced by leaky recombination in response to injury, we repeated the experiment in the absence of 4-OHT administration; we found no $\mathrm{GFP}^{+}$-recombined cells within the regenerated area (Supplementary Fig. $14 \mathrm{~g}-\mathrm{q} ; n=3 / 3$ ). We further excluded that the result is caused by 4 -OHT remaining in the body at the moment of injury through treating zebrafish with 4OHT 10 and 11 days before the injury (Supplementary Fig. 14r-w; $n=5 / 5)$. Taken together, our results reveal that trabeculae contribute to the regeneration of cortical myocardium. This process occurs concomitantly to the downregulation of $t b \times 5 a$ expression, suggesting that trabecular cardiomyocytes change their specification and become cortical cardiomyocytes.

The list of genes to discriminate cortical from trabecular myocardium in the zebrafish is short: besides the herein reported tbx5a expression pattern and $n p p a^{29}$, no other markers have been described to differentiate cortical from trabecular myocardium in the zebrafish. To interrogate whether $t b x 5 a$-derived trabecular cardiomyocytes not only relocate into the cortical region and switch off $t b \times 5 a$ but also adopt the expression of cortical marker genes, we performed RNA sequencing (RNA-Seq) comparing tbx5a-positive and -negative cardiomyocytes isolated from adult ventricles (Fig. 7a, b) . tbx5a was the top gene differentially expressed between the two populations (Fig. 7c and Supplementary Data 1), further supporting that the transgenic $t b x 5 a$ reporter lines reproduce the endogenous pattern. A number of genes related to actin cytoskeleton, extracellular matrix, and caveolae were expressed specifically in $t b \times 5 a^{-}$cardiomyocytes (Fig. $7 c, d$ ): two of these markers, xirp $2 a^{30}$ and lama5, were validated by immunofluorescence for their protein product (Figs. 8 and 9). In uninjured hearts, Xirp2a was detected in the cortical myocardium (Fig. $8 \mathrm{a}-\mathrm{f}, n=3 / 3$ ). Strikingly, the $t b x 5 a$-derived $\mathrm{GFP}^{+}$cells that contributed to the cortical layer and switched off $t b \times 5 a$ :mCherry also expressed Xirp2a (Fig. 8g-r, $n=6 / 6$ ). Laminin was detected in the cortical layer (Fig. 9a-f, $n=3 / 3$ ) and, upon injury, cortically 


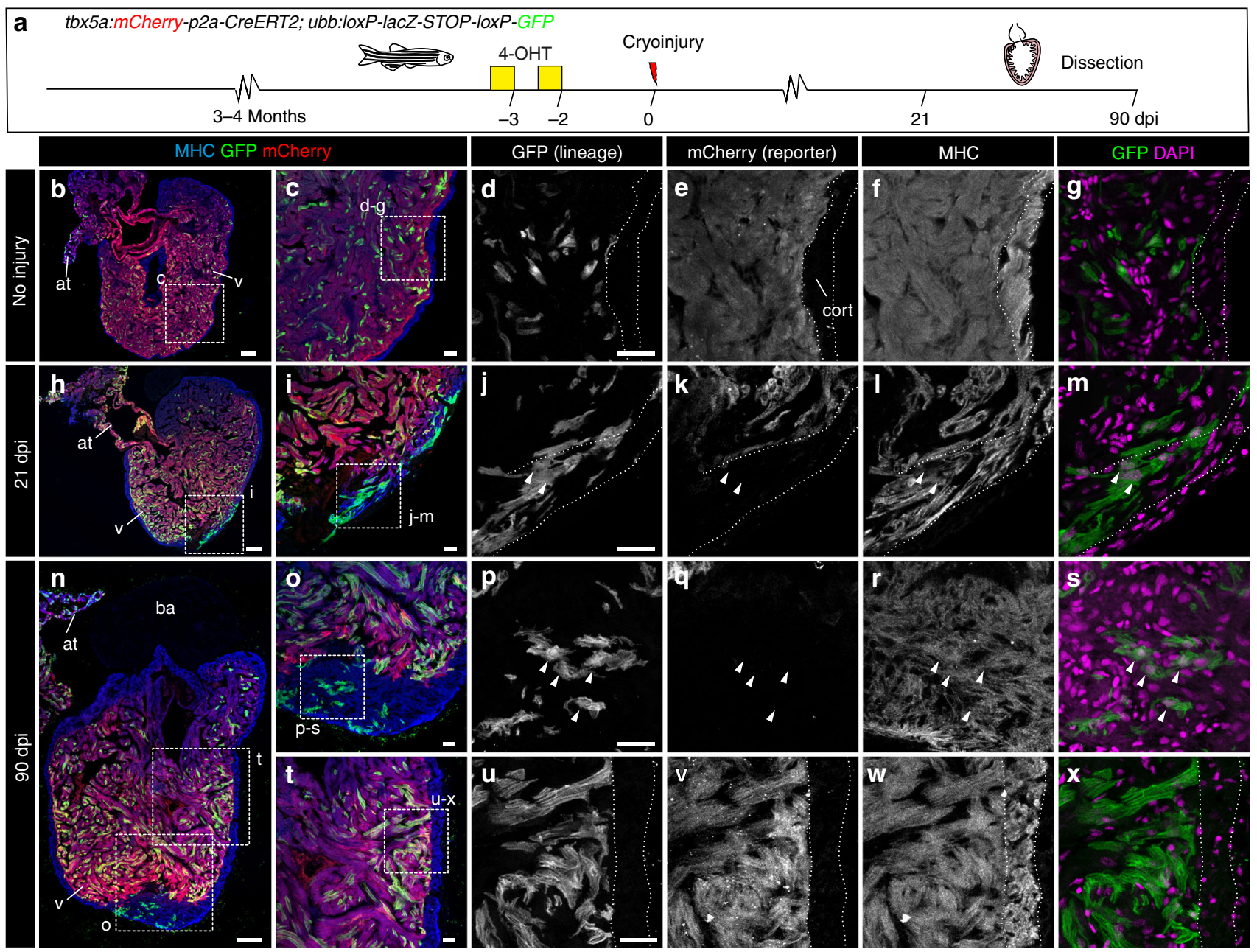

Fig. 6 Contribution of $t b \times 5 a$-derived cells during regeneration of the adult zebrafish heart. a tbx5a:Cherry-p2A-CreER ${ }^{T 2}$ was crossed into ubb:loxP-lacZ-STOPloxP-GFP. 4-Hydroxytamoxifen (4-OHT) was added 2 and 3 days before cryoinjury, to induce recombination of loxP sites. Hearts were fixed at 21 and 90 days postinjury (dpi) and sectioned for immunofluorescent detection of GFP ${ }^{+}$tbx5a-derived cells and mCherry ${ }^{+}$tbx5a-expressing cells. Nuclei were counterstained with DAPI. $\mathbf{b}$ In the uninjured heart $(n=7 / 7)$, mCherry expression was homogeneous in the trabecular myocardium and absent in the cortical layer. GFP ${ }^{+}$cells were found in the trabecular layer. c Zoomed view of boxed area in $\mathbf{b}$. $\mathbf{d}-\mathbf{f}$ Single channels of boxed area shown in c. $\mathbf{g}$ GFP and DAPI channels only. $\mathbf{h}, \mathbf{n}$ Section of hearts at $21 \mathrm{dpi} \mathbf{h}$ and $90 \mathrm{dpi} \mathbf{n}$. Upon cryoinjury to the ventricular apex, tbx5a-derived cardiomyocytes were present also in the cortical layer, particularly at the site of injury (h-m, $n=6 / 7 ; \mathbf{0}-\mathbf{s}, n=5 / 6 ; \mathbf{t}-\mathbf{x}, n=6 / 6)$, whereas tbx5 $a^{+}$cardiomyocytes in general were restricted to the trabecular myocardium $(n=5 / 6) \mathbf{t}-\mathbf{x}$. Nuclear counterstaining revealed GFP ${ }^{+}$cell bodies in the cortical layer (arrowheads). at, atrium; cort, cortical layer; ba, bulbus arteriosus; v, ventricle. Scale bars, b, h,n $100 \mu \mathrm{m}$ and $\mathbf{c}, \mathbf{d}, \mathbf{i}, \mathbf{j}, \mathbf{0}, \mathbf{p}, \mathbf{t}, \mathbf{u} 25 \mu \mathrm{m}$

located $t b x 5 a$-derived $\mathrm{GFP}^{+}$were in a Laminin ${ }^{+}$environment; Fig. 9g-r, $n=6 / 6$ ). This result was further supported using a RNAScope protocol to detect lama5 mRNA in situ (Supplementary Fig. 15; a-h, $n=4 / 4$; i-p, $n=11 / 11$ ). A third gene used to determine whether $t b \times 5 a$-derived cardiomyocytes express cortical markers when found in the cortical myocardium was hey2, a wellestablished marker of the compact layer in the embryonic mouse heart $^{31}$ (Supplementary Fig. 16). hey2 mRNA in situ detection by RNAScope revealed expression in cardiomyocytes from the cortical myocardium of the adult zebrafish (Supplementary Fig. 16 a-h; $n=3 / 4$ ). tbx5a-derived trabecular cardiomyocytes that were within the cortical region at $90 \mathrm{dpi}$ co-localized with hey 2 staining (Supplementary Fig. 16 i-m; $n=5 / 6$ ). Of note, $t b x 5 a$-derived cells within the trabecular myocardium did not reveal similar hey2 expression levels (Supplementary Fig. $16 n-p ; n=5 / 6$ ). On the contrary, the expression of the trabecular marker $n p p a$ was not retained in $t b x 5 a$-derived cells within the cortical myocardium (Supplementary Fig. 17, $n=7 / 7$ ), altogether indicating that trabecular $t b \times 5 a^{+}$cardiomyocytes undergo a true phenotypic switch to become cortical layer cardiomyocytes. Although the cortical myocardium had previously been reported to regenerate from cardiomyocytes within the same layer ${ }^{5}$, our results reveal that trabecular cardiomyocytes can also contribute to cortical layer repair in response to injury.

\section{Discussion}

In the developing hearts of birds and mammals, a border between Tbx5-positive and -negative cells determines the region of ventricular septation ${ }^{32}$. Our results here establish that the embryonic ventricle in zebrafish is concomitantly also split into $t b \times 5 a^{+}$and tbx $5 a^{-}$domains. A similar boundary between $d r l^{+}$and $d r l^{-}$cells has been described to separate the embryonic ventricle into domains to control distinct physiological properties ${ }^{11}$, suggesting that similar to mammals, adult teleost cardiac ventricles maintain FHF and SHF lineage territories (Fig. 10a). Although the zebrafish ventricle is not septated, the presence of a FHF-SHF-like 
a

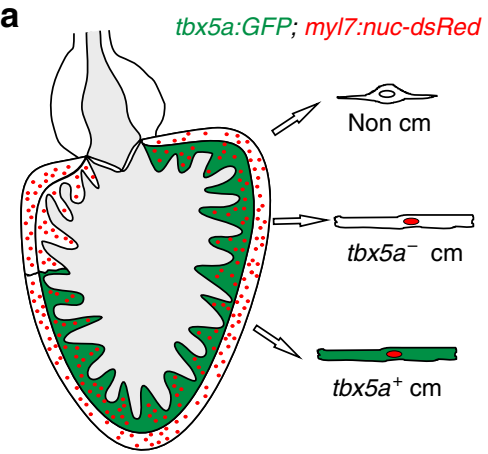

C

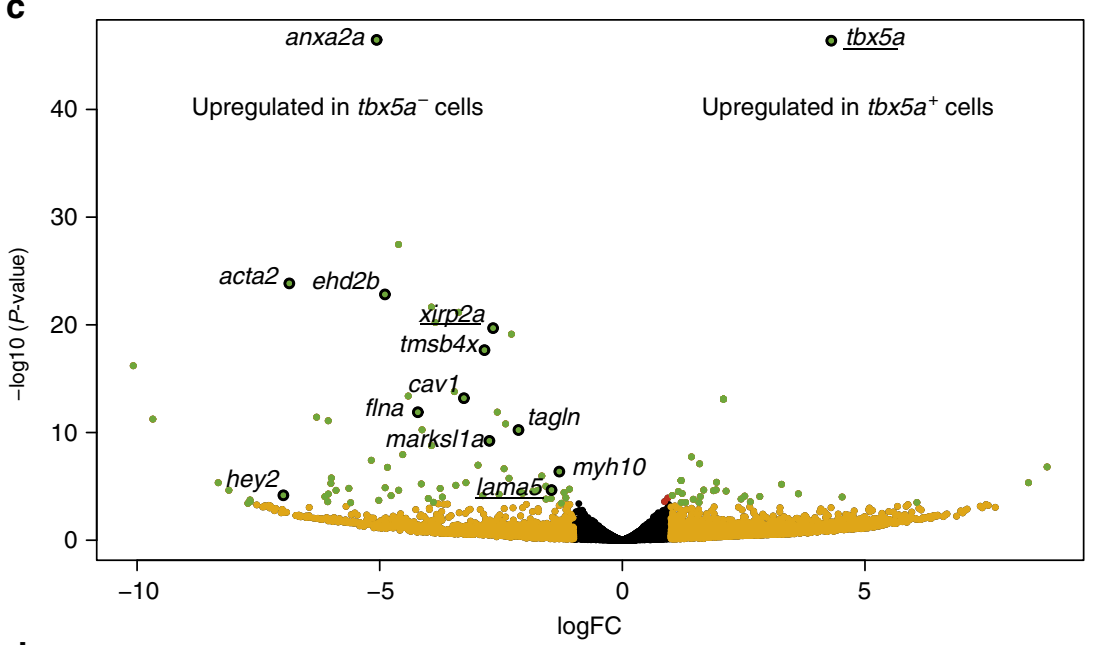

b

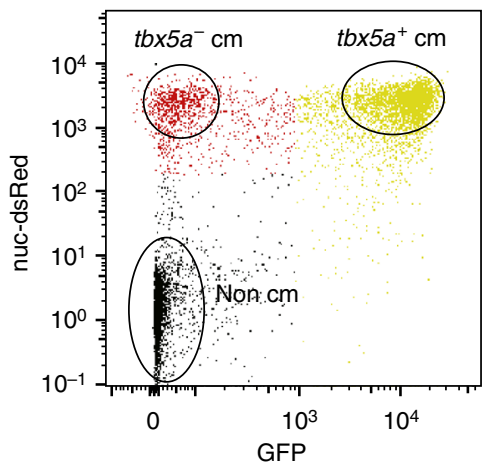

d

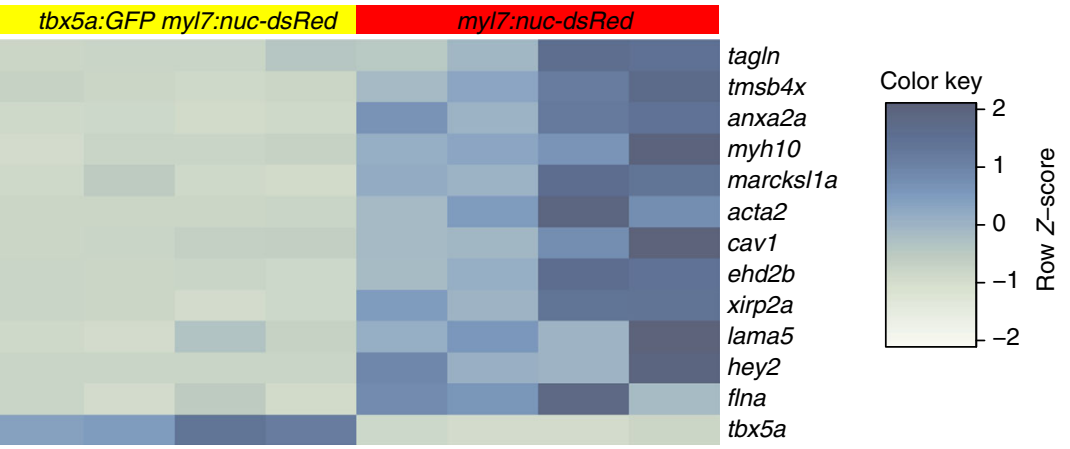

Fig. $7 \mathrm{tb} \times 5 \mathrm{a}^{+}$and $t b \times 5 a^{-}$cardiomyocytes from adult ventricles exhibit distinct expression profiles. a, $\mathbf{b} \mathrm{GFP}^{+} /$nuc-dsRed $^{+}$and $\mathrm{GFP}^{-} / \mathrm{nuc}^{-\mathrm{dsRed}}{ }^{+}$ cardiomyocytes were fluorescence-activated cell (FAC) sorted from adult tbx5a:GFP;myl7:nuc-dsRed ventricles $(n=5$ pooled heart per replicate; four replicates in total). c Volcano plot representing RNA-seq results comparing both populations. Black, false discovery rate (FDR) $>0.05$, log fold change (LFC) $<1$; orange, FDR $>0.05$, LFC $>1$; red, FDR $<0.05$, LFC $<1$; green, FDR $<0.05$, LFC $>1$. d Heatmap of genes differentially expressed in tbx5a and tbx $5 a^{-}$cardiomyocytes from adult hearts. Dark blue, higher expression; light blue, lower expression. cm, cardiomyocytes

boundary might represent a state that has been further progressed in evolution to allow morphogenetic events leading to septation.

Our results indicate that cardiomyocyte plasticity allows the ventricle to re-form exclusively from SHF-derived cardiomyocytes (Fig. 10b). Of note, in mammals, Tbx5 expression labels the derivatives of the FHF in the ventricle and also the boundary with the $\mathrm{SHF}^{19,20}$; thus, we cannot exclude that the contribution of the SHF is slightly bigger than described in our study. Nonetheless, our results show that SHF cardiomyocytes can compensate the loss of the whole FHF-derived ventricle.

tbx $5 a$ lineage tracing allowed (i) to confirm that the cortical layer forms during development from the trabecular myocardium $^{5}$ and (ii) to identify that this process involves not only a change in location of cardiomyocytes from the trabecular to the cortical regions but also a change in gene expression (Fig. 10a).

In contrast to development, cardiomyocyte plasticity seems to be reduced in the regenerating adult heart: ${ }^{19}$ conversion of atrial cardiomyocytes to ventricular cardiomyocytes has not been conclusively reported ${ }^{7}$ and Gupta et al. ${ }^{5}$ described that the newly regenerated cortical layer derives from pre-exising cortical layer cardiomyocytes. Indeed, the general rule during regeneration is that every cell contributes to the same cell type it was before injury, as illustrated by the cell types comprising the axolotl $\operatorname{limb}^{33}$. In contrast, our results show that a breach of this behavior is possible, with trabecular cardiomyocytes re-specialising into cortical layer cardiomyocytes during regeneration of the adult zebrafish heart (Fig. 10c). This re-specialisation reuses a 

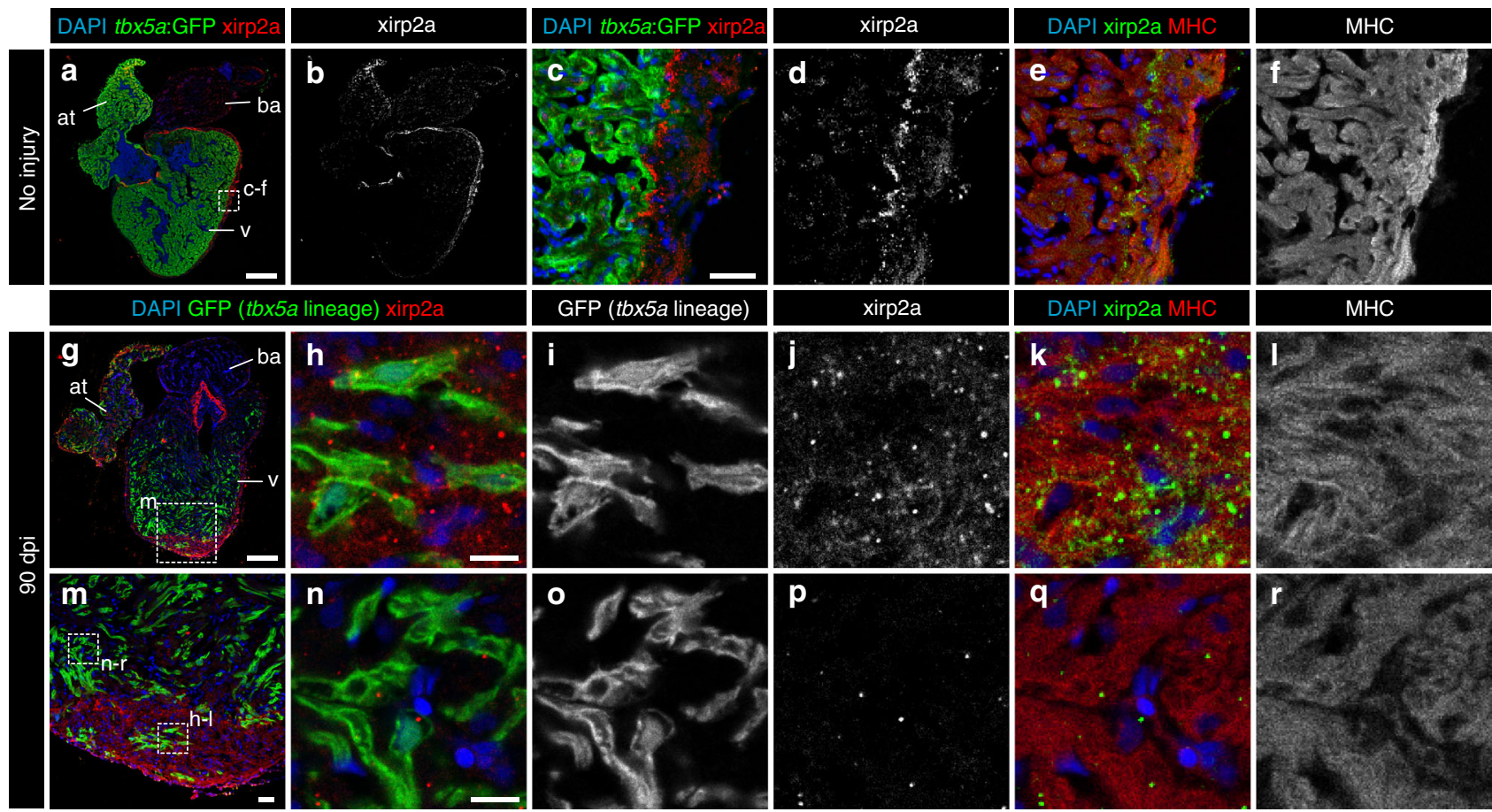

Fig. 8 Trabecular tbx5a-derived cardiomyocytes within the cortical myocardium express the cortical marker Xirp2a. Immunofluorescence with anti-Xirp2a and GFP and anti-Myosin Heavy Chain (MHC) on ventricle sections. a-f Uninjured adult tbx5a:GFP ventricle. Xirp2a expression was observed in the cortical layer but not in the trabecular layer showing a complementary pattern with $t b x 5 a$ :GFP as predicted by the RNA-Seq $(n=3 / 3)$. g-r Double transgenic tbx5a: mCherry-p2a-CreER ${ }^{T 2}$; ubb:loxP-lacZ-loxP-GFP were treated with 4-Hydroxytamoxifen (4-OHT) from 84 to 72 and 60 to 48 hours before cryoinjury. Hearts were fixed at 90 days postinjury (dpi). GFP ${ }^{+}$cells marking the $t b x 5 a$ lineage within the cortical layer were positive for Xirp2a, whereas GFP ${ }^{+}$cells within the trabecular layer did not express this marker $(n=6 / 6)$. at, atrium; ba, bulbus arteriosus; $v$, ventricle. Scale bars, $\mathbf{a}, \mathbf{g} 100 \mu \mathrm{m}, \mathbf{c}, \mathbf{m} 25 \mu \mathrm{m}$, and $\mathbf{h}, \mathbf{n} 10 \mu \mathrm{m}$

developmental process in the setting of regeneration to rebuild the injured heart. It is interesting to note that zebrafish hearts are highly trabeculated in comparison with adult mammals. In murine cardiogenesis, the trabecular myocardium is proliferating at a much lower rate than the compact myocardium ${ }^{34}$. Thus, the switch from a trabecular phenotype to cortical phenotype might additionally allow the cardiomyocytes to re-enter a more active rate of cycling, allowing them to drive the regeneration after cryoinjury in the adult context. We propose that cardiomyocyte plasticity is thus likely a key feature necessary to react efficiently with a regenerative response to injury.

\section{Methods}

Animal handling and generation of transgenic lines. Ethical approval was obtained from the Community of Madrid "Dirección General de Medio Ambiente" in Spain and the "Amt für Landwirtschaft und Natur" from the Canton of Bern, Switzerland. Animals were housed and experiments performed in accordance with Spanish and Swiss bioethical regulations for the use of laboratory animals. Fish were maintained at a water temperature of $28^{\circ} \mathrm{C}$.

The construct to generate $\operatorname{Tg}(t b x 5 a: t d$ Tomato $)$ transgenic zebrafish lines was made by recombining the tdTomato cassette (Supplementary Data 2) into the BAC CH73-99A14. The construct for $T g\left(t b x 5 a: m C h e r r y-p 2 A-C r e E R^{T 2}\right)^{\mathrm{cn} 4}$ was generated by recombining the $i$ Tol2Amp cassett ${ }^{35}$ (Supplementary Table 1, primers 1,2) and $m$ Cherry-p2A-CreER ${ }^{T 2}$ (Supplementary Data 3 and Supplementary Table 1, primers 3,4$)$ into the same BAC. The construct to generate $\operatorname{Tg}\left(\text { tbx } 5 a: C r e E R^{T 2}\right)^{\mathrm{cn} 3}$ was made by recombining iTol2Amp- $\gamma$-crystallin:RFP (Supplementary Data 4 and Supplementary Table 2, primers 2,5) and $\mathrm{CreER}^{\mathrm{T} 2}$ (Supplementary Table 1; primers 4,6) cassettes into the same BAC. Tg(vmhcl:loxP-myctagBFP-STOP-loxP$N T R-m$ Cherry $)^{\mathrm{cn} 5}$ was generated using a construct obtained from recombining $i$ Tol2 Amp $^{35}$ (Supplementary Table 1, primers 1,2) and loxP-myctagBFP-STOPloxP- $m$ Cherry-NTR (Supplementary Data 5 and Supplementary Table 2, primers 7,8) cassettes into the BAC CH73-204E19. Plasmid templates for recombineering were cloned using Gibson Assembly (NEB). Recombineering was performed combining the pRedET (GeneBridges, Germany) system and EL250 bacteria ${ }^{36} . T g$ (-3.5ubb:loxP-lacZ-loxP-eGFP) cn2 26 was outcrossed for six generations to isolate the best insertion. BAC DNA was injected at $25 \mathrm{ng} \mathrm{\mu l}^{-1}$ into one-cell stage zebrafish embryos along with $1 \mathrm{nl}$ of $50 \mathrm{ng} \mathrm{\mu l}^{-1}$ synthetic Tol2 mRNA in Danieau buffer. Transient ltbp3:TagRFP-2A-Cre embryos in the stable tbx5a:GFP background were generated by injecting a Tol2 plasmid containing the vector ltbp3:TagRFP-p2A$C r e^{12}$ into one-cell stage $\operatorname{Tg}(t b x 5 a: G F P)$ embryos. Around 150 embryos survived the microinjection and were screened for mCherry expression. The transgenic line

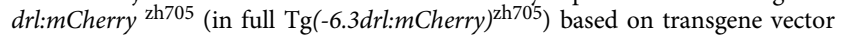
pCM330 was generated using Multisite Gateway assembly of pCM293 (pENTR5' backbone containing $6.35 \mathrm{~kb}$ of the zebrafish $d r l$ locus (ZDB-GENE-991213-3) amplified with primers $5^{\prime}$-GTCAGCACCAGATGCCTGTGC-3' (forward) and $5^{\prime}$ CCAAGTGTGAATTGGGATCG-3' (reverse) as described ${ }^{37}$ ), Tol2kit ${ }^{38} \# 386$ (pME$m$ Cherry), \#302 (p3E_SV40polyA), and \#394 (pDestTol2A2) (in total pDestTol2pA2_drl:mCherry, referred to as drl:mCherry); plasmid DNA was injected at $25 \mathrm{ng} \mathrm{\mu l}^{-1}$ into one-cell stage zebrafish embryos that were then raised and screened for germline transmission of the transgenic reporter with subsequent outcrossing to isolate a single transgene insertion ${ }^{39}$. The drl:mCherry transgenics used in the study are at least seventh-generation transgenics. The line $\mathrm{Tg}(-26.5 \mathrm{Hsa}$. WT1-gata2:EGFP) $)^{\mathrm{cn} 1} 40$ contains a reporter construct flanked by flippase recognition target sites, in which cardiac actin drives the expression of RFP. This cassette was removed by injection of flippase mRNA into the one-cell stage. This line was called $\operatorname{Tg}(-26.5 H s a \text {.WT1-gata2:EGFP })^{\mathrm{cn} 12}$ and was replacing Tg $(-26.5 H s a . \text { WT1-gata2:EGFP })^{\mathrm{cn} 1}$.

In adults, $10 \mu \mathrm{M}$ 4-OHT (Sigma, H7904) was administered at the indicated times. Treatments were performed overnight. Before administration, the $10 \mathrm{mM}$ stock (dissolved in ethanol) was heated $10 \mathrm{~min}$ at $65^{\circ} \mathrm{C}^{39}$. For genetic labeling in tbx5a:mCherry-p2A-CreER $R^{T 2}$;3.5ubb:loxP-lacZ-loxP-eGFP embryos, 4-OHT was administered at $10 \mu \mathrm{M}$ from 24 to $48 \mathrm{hpf}$ and at $5 \mu \mathrm{M}$ from 48 to $84 \mathrm{hpf}$. Cryoinjury was performed as previously described using a copper filament cooled in liquid nitrogen and placed on the ventricular surface of the heart until thawing ${ }^{41}$. The pericardial cavity had been previously opened to expose the heart, in anesthetized animals. For genetic ablation experiments and their controls, 4-OHT was administered at $5 \mu \mathrm{M}$ from 24 to $48 \mathrm{hpf}$ and then Mtz (Sigma, M3761) was added at $10 \mathrm{mM}$ from 96 to $168 \mathrm{hpf}$. Other lines used were TgBAC(tbx5a:GFP)in2Tg; ${ }^{24}$ $\operatorname{Tg}($ myl7:nDsRed $) \mathrm{f}^{42}{ }^{2} \mathrm{Tg}(\text { myl7:membranemCherry })^{43}, \mathrm{Tg}(3.5$ ubb:loxP-mCherryloxP-eGFP $)^{44}$, and $\mathrm{Et}(-26.5 \mathrm{Hsa} \text {.WT1-gata2:EGFP })^{\mathrm{cn} 1}(\text { epi:GFP })^{40}$

For all the experiments involving Cre recombination or cell ablation, hemizygous fish were used. For experiments using only the $\operatorname{Tg}(t b x 5 a: G F P)$ line, homozygous and hemizygous fish were used interchangeably.

BrdU was added to $\mathrm{E} 3$ water at $5 \mathrm{mg} \mathrm{ml}^{-1}$ with $0.5 \%$ dimethyl sulfoxide from 4 to $7 \mathrm{dpf}$. 

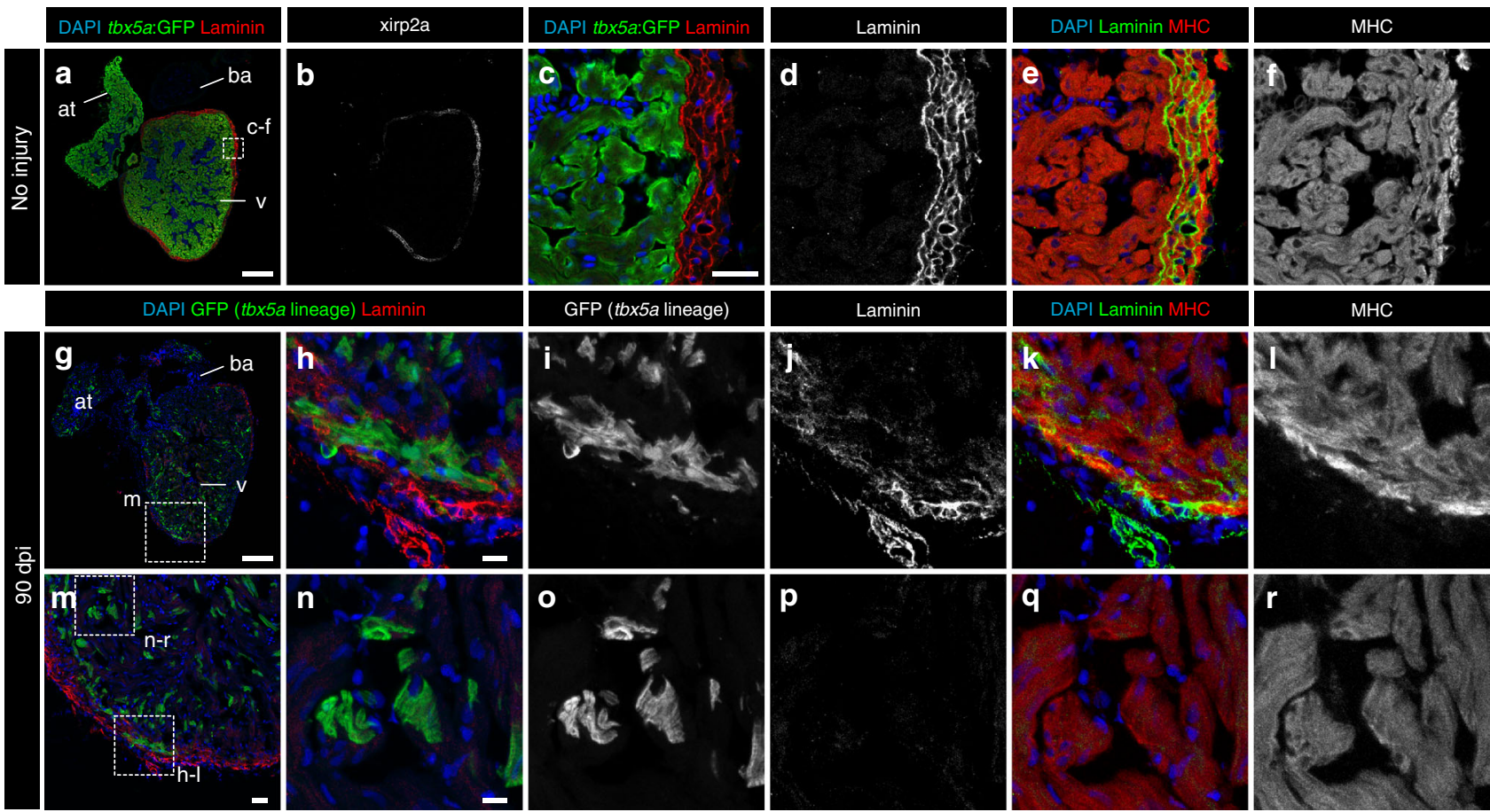

Fig. 9 Trabecular tbx5a-derived cardiomyocytes within the cortical myocardium are surrounded by Laminin. Immunofluorescence with anti-Laminin, antiGFP, and anti-Myosin Heavy Chain (MHC) on ventricle sections. a-f Uninjured adult tbx5a:GFP ventricle. Laminin expression was observed in the cortical layer but not in the trabecular layer showing a complementary pattern with tbx5a:GFP $(n=3 / 3)$. g-r Double transgenic tbx5a:mCherry-p2a-CreER ${ }^{T 2}$; $u b b$ :loxPlacZ-loxP-GFP adult fish were treated with 4-Hydroxytamoxifen (4-OHT) from 84 to 72 and 60 to 48 hours before cryoinjury. Hearts were fixed at 90 days postinjury (dpi). GFP cells marking the tbx5a lineage within the cortical layer were surrounded by Laminin staining $(n=6 / 6)$. at, atrium; ba, bulbus arteriosus; $v$, ventricle. Scale bars, $\mathbf{a}, \mathbf{g} 100 \mu \mathrm{m}, \mathbf{c}, \mathbf{m} 25 \mu \mathrm{m}$, and $\mathbf{h}, \mathbf{n} 10 \mu \mathrm{m}$

Histology. Samples for Fig. 2a Fig. 3a, and Supplementary Figures 1c-g, 3, 4, 13 and 17 were fixed in $4 \%$ pParaformaldehyde (PFA) in phosphate-buffered saline (PBS) overnight at $4{ }^{\circ} \mathrm{C}$. Samples were then washed in $0.1 \%$ Tween 20 (Merck) in PBS, dehydrated through an ethanol series, and embedded in paraffin wax. Samples were sectioned at $7 \mu \mathrm{m}$ with a microtome (Leica), sections mounted on Superfrost slides (Fisher Scientific), and dried overnight at $37^{\circ} \mathrm{C}$.

The rest of sections were obtained by fixing in $4 \%$ PFA washing in PBS $+0.1 \%$ Tween 20 (PBT), incubated in $15 \%$ saccharose overnight $4{ }^{\circ} \mathrm{C}$. Then, samples were embedded in $30 \%$ gelatin $15 \%$ saccharose and snap frozen at $-80^{\circ} \mathrm{C}$ in isopentane. Tissue was cut at $8 \mu \mathrm{m}$ on a cryostat (Leica).

In situ mRNA hybridization. In situ mRNA hybridisation on paraffin sections and on whole-mount larvae were performed as described ${ }^{45,46}$ using $t b x 5 a$ (complementary DNA kindly provided by C. Neumann), GFP (cDNA kindly provided by J.L. Gómez-Skarmeta), and $n p p a^{47}$ riboprobes.

Paraffin sections were deparaffinized, postfixed $20 \mathrm{~min}$ with PFA $4 \%$, washed with PBS, treated with proteinase $\mathrm{K} 10 \mu \mathrm{m} \mathrm{ml}^{-1}$ for $10 \mathrm{~min}$ at $37^{\circ} \mathrm{C}$, washed with PBS, postfixed with PFA $4 \%$ for 5 min, washed with PBS, treated with $\mathrm{HCl} 0.07 \mathrm{~N}$ for $15 \mathrm{~min}$, washed with PBS, treated with $0.25 \%$ acetic anhydride in triethanolamine $0.1 \mathrm{M} \mathrm{pH} 8$ for $10 \mathrm{~min}$, washed with PBS, washed with RNase-free water, and then hybridised with the probe in pre-hybridisation buffer over night at $65^{\circ} \mathrm{C}$. The following day, sections were washed twice with post-hybridisation buffer $1(50 \%$ formamide, $5 \times \mathrm{SSC}, 1 \% \mathrm{SDS})$ for $30 \mathrm{~min}$ at $65^{\circ} \mathrm{C}$ and twice more with post-hybridisation buffer 2 ( $50 \%$ formamide, $2 \times$ saline-sodium citrate SSC, $1 \%$ SDS). Then, sections were washed with maleic acid buffer with $0.1 \%$ Tween 20 (MABT) buffer at room temperature and incubated at least $2 \mathrm{~h}$ in blocking solution at room temperature. They were next incubated overnight with anti-digalkaline phosphatase (AP) antibody $(1: 2,000)$ in blocking solution. The third day, they were washed with MABT for several hours, followed by washes in AP buffer. Sections were developed in BM-Purple (Roche) until signal was detected.

For whole-mount in situ hybridisations, embryos were fixed in 4\% PFA overnight, dehydrated in methanol series, and stored at $-20^{\circ} \mathrm{C}$ until its use. On day 1, embryos were rehydrated, washed in PBT, digested with $10 \mu \mathrm{g} \mathrm{m}^{-1}$ for 15 $\mathrm{min}$, rinsed in PBT, and fixed in 4\% PFA for $10 \mathrm{~min}$. After washing again in PBT, they were pre-hybridised at $67^{\circ} \mathrm{C}$ for at least $1 \mathrm{~h}$. The antisense riboprobe was added at $0.5 \mathrm{\mu g} \mathrm{ml}^{-1}$. After overnight hybridisation, two washes with $50 \%$ formamide $/ 2 \times$ SSCT, one wash with $2 \times$ SSCT, and an additional two washes with $0.2 \times$ SSCT were performed, all at $67^{\circ} \mathrm{C}$. Then, embryos were transferred to room temperature and washed in MABT. Antibody incubation, washing, and color development condition times were as for in situ on sections. RNAScope (Advanced
Cell Diagnostics, Hayward, CA) was performed following the manufacturer's instructions for formalin-fixed paraffin-embedded samples with standard tissue pretreatment and 2.5 HD RED detection kit.

Immunofluorescence and TUNEL in whole-mount embryos. Embryos were fixed in $4 \%$ PFA overnight, washed in $0.1 \%$ Tween 20 in PBS, and permeabilised with $0.5 \%$ Triton X-100 (Sigma) in PBS for 20 min. Several washing steps were followed by $2 \mathrm{~h}$ of blocking with $5 \%$ goat serum, $5 \%$ bovine serum albumin (BSA), and 20 $\mathrm{mM} \mathrm{MgCl} 2$ in PBS, followed by incubation with antibodies overnight. Apoptosis was detected by TUNEL staining using the in situ cell death detection kit from Roche (Mannheim, Germany), incubating fish embryos in enzyme solution for $1 \mathrm{~h}^{48}$.

Immunofluorescence on sections. Paraffin sections were deparaffinized, rehydrated, and washed in distilled water. Epitopes were retrieved by heating in $10 \mathrm{mM}$ citrate buffer ( $\mathrm{pH}$ 6.0) for $15 \mathrm{~min}$ in a microwave at full power. Gelatine sections were incubated instead $30 \mathrm{~min}$ in $0.1 \%$ Tween 20 in PBS at $37^{\circ} \mathrm{C}$ to dissolve the gelatin. Nonspecific binding sites were saturated by incubation for at least $1 \mathrm{~h}$ in blocking solution (5\% BSA, $5 \%$ goat serum, and $20 \mathrm{mM} \mathrm{MgCl}_{2}$ in PBS). Endogenous biotin was blocked with the avidin-biotin blocking kit (Vector, Burlingame, CA, USA).

Antibodies. Primary antibodies used were anti-Myosin Heavy Chain (MF20, DSHB, $1: 20$ and F59, DSHB, $1: 20$ ), anti-GFP (AVES, GFP-1010, $1: 500 ; 632592$, Clontech, Mountain View, CA, USA, $1: 100$ ), anti-RFP (ab34771, AbCam, $1: 200$ ), anti-Xirp2 $\mathrm{a}^{30}$, a kind gift from C. Otten and S. Seyfried $(1: 500)$, anti-Laminin (L9393, Sigma, $1: 200$ ), anti-BrdU (BD Biosciences, B44, $1: 100$ ), anti-mKate ${ }^{49}$ to detect tagBFP $(1: 500)$. Biotin- or Alexa $(488,568,633)$-conjugated secondary antibodies, and streptavidin-Cy3 (Jackson Immuno Research Laboratories) were used at $1: 300$. Nuclei were stained with 4',6-Diamidino-2-phenylindole (DAPI) and slides were mounted in Fluorsave (Calbiochem).

Imaging and Image analysis. Embryos were imaged with a Zeiss 780 confocal microscope using a $\times 20$ objective with a dipping lens. $Z$-stacks were taken every 1 $\mu \mathrm{m}$. Three-dimensional images were reconstructed with ImageJ software. A Leica TCS SP-5 or a Nikon A1R confocal microscope was used for imaging of histological sections. The percentage of tagBFP cells was quantified using ImageJ considering the area of tagBFP and comparing it with the area of myosin heavy chain (MHC) staining. 


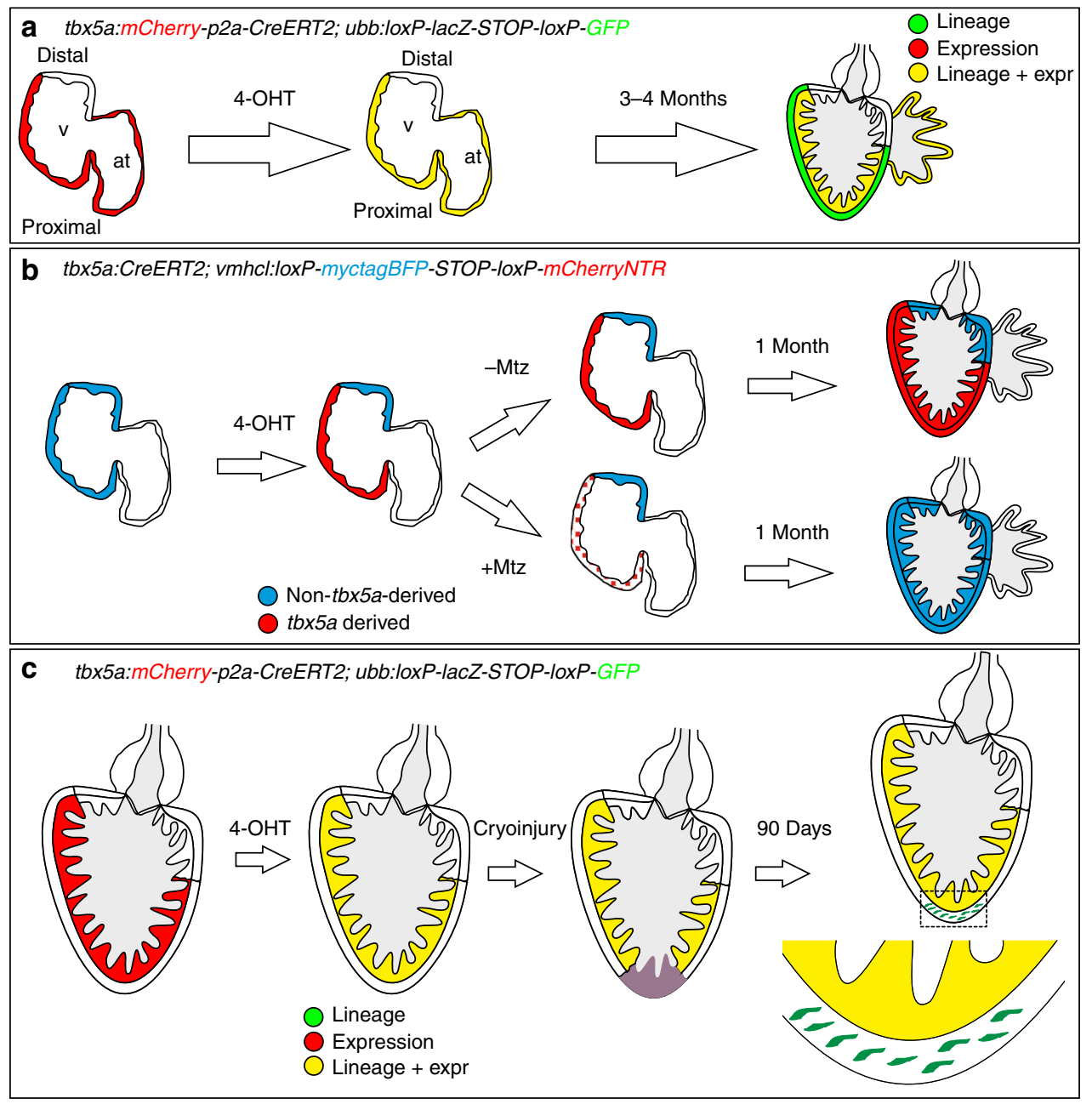

Fig. 10 Summary of the contribution of tbx5a-positive and -negative myocardium during heart development and regeneration. a Identification of tbx5aexpressing and tbx5a-derived cells in the zebrafish heart. Red, tbx $5 a^{+}$cells; green, tbx5a-derived cells not expressing tbx5a; yellow, tbx5a-expressing cells derived from embryonic $t b \times 5 a^{+}$cells. $\mathbf{b}$ Replacement of the embryonic first heart field myocardium with second heart field progenitors. Blue, ventricular cardiomyocytes; red, tbx5a-derived ventricular cardiomyocytes; dotted red, ablated tbx5a-derived ventricular cardiomyocytes. c Contribution of tbx5aderived cells during heart regeneration in the zebrafish. Red, $t b \times 5 a^{+}$cells; green, tbx5a-derived cells not expressing tbx5a; yellow, tbx5a-expressing cells derived from trabecular adult tbx5a+ cells. 4-OHT, 4-Hydroxytamoxifen; at, atrium; Mtz, Metronidazole; v,ventricle

The percentage of $t b x 5 a$-derived cells was quantified applying a median filter of radius 1 in ImageJ. The $\mathrm{GFP}^{+}$and $\mathrm{MHC}^{+}$areas were measured. The same set of images was used to quantify the percentage of $\mathrm{GFP}^{+}$cardiomyocytes in trabeculae and in the regenerated compact layer, applying the same threshold for both regions.

In vivo imaging and image processing. Double transgenic $\operatorname{Tg}(t b x 5 a: t d T o m a t o) ; \mathrm{Et}$ $(-26.5 \mathrm{Hsa}$.WT1-gata2:EGFP) lines were grown until $60 \mathrm{hpf}$ in E3 culture medium with $0.0033 \%$ 1-Phenyl-2thiourea. Embryos were anesthetized with $0.08 \mathrm{mg} \mathrm{ml}^{-1}$ Tricaine and embedded in $1 \%$ low melting agarose (Bio-Rad Low Melt Agarose). Confocal imaging of the embryonic heart was performed with a Zeiss LSM880, using a $\times 20 / 0.8$ air-objective lens. The green (GFP) and red (tdTomato) channels were acquired simultaneously with maximum speed in bidirectional mode. $Z$ stacks were acquired over $8 \mathrm{~h}$ with $10 \mathrm{~min}$ time interval. The range of detection for each channel was adapted to avoid any crosstalk between the channels. A median filter was applied to a representative frame to show co-expression of the epicardial marker and $t b x 5 a$.

Ultrasound and assessment of cardiac function in larvae. Measurements and analysis were performed as described ${ }^{47}$. A mixture of $60 \mathrm{mM}$ of tricaine and $3 \mathrm{mM}$ of isofluorane were added to the tank water in order to anesthetize the animals. A Petri dish was filled with this solution and the fish was placed ventral side up in a foam holder. Once the animal was anesthetised, the images were obtained with a VEVO 2100 system (VisualSonics) coupled to a $50 \mathrm{MHz}$ ultrasound transducer. The probe was lowered until an ultrasound conductive film was formed with the water, so that the skin of the animal was not touched at any time. This way the animal position was rotated till we obtained a long axis view of the heart with a complete visualisation of the ventricular apex. In that moment we recorded cine two-dimensional sequences, with a frame rate of 200 images per second, which were analysed later, in a second step, to minimize anesthesia times.

Imaging of cardiac function in larvae was performed using a Leica AM TIRF MC microscope in epifluorescence mode, using an Andor DU 885K-CS0-\#VP $(1,004 \times 1,002)$ camera and HCX PL APO $20 \times 0,7$ dry $(11,506,166)$ objective. The maximum (diastolic) and minimum (systolic) ventricular areas were measured in order to determine ventricular function using Image J.

Heart dissociation followed by sorter and RNA-Seq library production. Zebrafish hearts were dissected and the atrio-ventricular canal was carefully removed in order to obtain a pool of exclusively ventricular cells. The ventricles were dissociated according to previous protocols ${ }^{50}$ with minor modifications. The enzyme concentration was doubled and time of digestion increased to $1 \mathrm{~h}$ and $40 \mathrm{~min}$ with gentle agitation while pipetting with a cut $1,000 \mu \mathrm{l}$ tip every $20-30 \mathrm{~min}$. Then, one volume of PBS $+10 \%$ fetal bovine serum (FBS) was added and the mixture was centrifuged for $8 \mathrm{~min}$ at $250 \mathrm{~g}$ and re-suspended in PBS $+1 \%$ FBS. Specifically, the following enzyme concentrations were used: liberase TH (Sigma, $200 \mathrm{mgl}^{-1}$ ), Elastase (Serva, $1: 250$ ), Pronase E (Serva, $1: 100$ dilution of the $26.3 \mathrm{mg} \mathrm{m}^{-1}$ stock), DNase (Qiagen, $1: 20,000$ ) and 2,3-butanedione monoxime (Sigma, 10 $\mathrm{mM}$ ). All reagents were dissolved in Tyrode's low calcium (in $\mathrm{mM}$ ): $\mathrm{NaCl} 140, \mathrm{KCl}$ 5.4, $\mathrm{CaCl}_{2} 0.01, \mathrm{MgCl}_{2} 1.0$, glucose 5.5, and HEPES 5.0; $\mathrm{pH}$ was set to 7.4 with $\mathrm{NaOH}$.

Cells were sorted using a SONY Synergy sy3200 sorter and RNA was extracted using the Arcturus Pico Pure RNA isolation kit (Thermofisher) following the manufacturer's instructions. 
Total RNA ( $0.6 \mathrm{ng})$ was used to generate barcoded RNA-Seq libraries using the Ovation Single Cell RNA-Seq System (NuGEN) with two rounds of library amplification. The size of the libraries was calculated using the Agilent 2100 Bioanalyzer. Library concentration was determined using the Qubit fluorometer (ThermoFisher Scientific). Libraries were sequenced on a HiSeq2500 (Illumina) platform to generate 60-base single reads. FastQ files for each sample were obtained using CASAVA v1.8 software (Illumina). Four biological replicates consisting of five pooled hearts were used per sample.

RNA-Seq analysis. Sequencing adaptor contaminations were removed from reads using cutadapt 1.9.1 software ${ }^{51}$ and the resulting reads were mapped and quantified on the transcriptome (Ensembl gene-build 10, release 82) using RSEM v1.2.25 $5^{52}$. Only genes with at least one count per million in at least two samples were considered for statistical analysis. Data were then normalised and differential expression tested using the bioconductor package Edge $^{53}$. We considered as differentially expressed those genes with a Benjamini-Hochberg adjusted $p$-value $\leq$ 0.05 and $\log$ fold change $\geq 1$. A heatmap was made using gplot library and heatmap.2 function.

Data availability. RNA-Seq data have been deposited in the GEO database under accession code GSE87596. The authors declare that all data supporting the findings of this study are available within the article and its supplementary information files or from the corresponding author upon reasonable request. Raw data corresponding to the Figures are deposited at Mendeley: https://doi.org/10.17632/ fkm2tvh2sv.1; https://doi.org/10.17632/4fp5f8t7j3.1 and https://doi.org/10.17632/ zgdff55m 44.1

Received: 10 April 2017 Accepted: 15 December 2017

Published online: 30 January 2018

\section{References}

1. Kikuchi, K. et al. Primary contribution to zebrafish heart regeneration by gata 4 (+) cardiomyocytes. Nature 464, 601-605 (2010).

2. Jopling, C. et al. Zebrafish heart regeneration occurs by cardiomyocyte dedifferentiation and proliferation. Nature 464, 606-609 (2010).

3. Gonzalez-Rosa, J. M., Burns, C. E. \& Burns, C. G. Zebrafish heart regeneration: 15 years of discoveries. Regeneration (Oxf.) 4, 105-123 (2017).

4. Liu, J. et al. A dual role for ErbB2 signaling in cardiac trabeculation. Development 137, 3867-3875 (2010).

5. Gupta, V. \& Poss, K. D. Clonally dominant cardiomyocytes direct heart morphogenesis. Nature 484, 479-484 (2012).

6. Gupta, V. et al. An injury-responsive gata4 program shapes the zebrafish cardiac ventricle. Curr. Biol. 23, 1221-1227 (2013).

7. Zhang, R. et al. In vivo cardiac reprogramming contributes to zebrafish heart regeneration. Nature 498, 497-501 (2013).

8. de Pater, E. et al. Distinct phases of cardiomyocyte differentiation regulate growth of the zebrafish heart. Development 136, 1633-1641 (2009).

9. Hami, D., Grimes, A. C., Tsai, H. J. \& Kirby, M. L. Zebrafish cardiac development requires a conserved secondary heart field. Development 138 , 2389-2398 (2011).

10. Lazic, S. \& Scott, I. C. Mef2cb regulates late myocardial cell addition from a second heart field-like population of progenitors in zebrafish. Dev. Biol. 354, 123-133 (2011)

11. Mosimann, C. et al. Chamber identity programs drive early functional partitioning of the heart. Nat. Commun. 6, 8146 (2015).

12. Zhou, Y. et al. Latent TGF-beta binding protein 3 identifies a second heart field in zebrafish. Nature 474, 645-648 (2011).

13 Buckingham, M., Meilhac, S. \& Zaffran, S. Building the mammalian heart from two sources of myocardial cells. Nat. Rev. Genet. 6, 826-835 (2005).

14 Waardenberg, A. J., Ramialison, M., Bouveret, R. \& Harvey, R. P. Genetic networks governing heart development. Cold Spring Harb. Perspect. Med. 4, a013839 (2014).

15 Spater, D. et al. A HCN4 +cardiomyogenic progenitor derived from the first heart field and human pluripotent stem cells. Nat. Cell. Biol. 15, 1098-1106 (2013).

16 Moretti, A. et al. Multipotent embryonic isl1 + progenitor cells lead to cardiac, smooth muscle, and endothelial cell diversification. Cell 127, 1151-1165 (2006).

17 Lescroart, F. et al. Early lineage restriction in temporally distinct populations of Mesp1 progenitors during mammalian heart development. Nat. Cell. Biol. 16, 829-840 (2014).

18 Bruneau, B. G. et al. Chamber-specific cardiac expression of Tbx 5 and heart defects in Holt-Oram syndrome. Dev. Biol. 211, 100-108 (1999).

19 Steimle, J. D. \& Moskowitz, I. P. TBX5: A key regulator of heart development. Curr. Top. Dev. Biol. 122, 195-221 (2017).

20 Devine, W. P., Wythe, J. D., George, M., Koshiba-Takeuchi, K. \& Bruneau, B. G. Early patterning and specification of cardiac progenitors in gastrulating mesoderm. Elife 3, (2014).
21 Albalat, R., Baquero, M. \& Minguillon, C. Identification and characterisation of the developmental expression pattern of tbx $5 \mathrm{~b}$, a novel tbx 5 gene in zebrafish. Gene Expr. Patterns 21, 24-30 (2010).

22 Garrity, D. M., Childs, S. \& Fishman, M. C. The heartstrings mutation in zebrafish causes heart/fin Tbx5 deficiency syndrome. Development 129, 4635-4645 (2002).

23 Pi-Roig, A., Martin-Blanco, E. \& Minguillon, C. Distinct tissue-specific requirements for the zebrafish tbx5 genes during heart, retina and pectoral fin development. Open Biol. 4, 140014 (2014).

24 Ocana, O. H. et al. A right-handed signalling pathway drives heart looping in vertebrates. Nature 549, 86-90 (2017).

25 Goldman, J. A. et al. Resolving heart regeneration by replacement histone profiling. Dev. Cell 40, 392-404 e395 (2017).

26 Di Donato, V. et al. 2C-Cas9: a versatile tool for clonal analysis of gene function. Genome Res. 26, 681-692 (2016).

27 Singh, A. R. et al. Chamber specific gene expression landscape of the zebrafish heart. PLoS ONE 11, e0147823 (2016).

28 Curado, S., Stainier, D. Y. \& Anderson, R. M. Nitroreductase-mediated cell/ tissue ablation in zebrafish: a spatially and temporally controlled ablation method with applications in developmental and regeneration studies. Nat. Protoc. 3, 948-954 (2008).

29 Jensen, B. et al. Identifying the evolutionary building blocks of the cardiac conduction system. PLoS. ONE 7, e44231 (2012).

30 Otten, C. et al. Xirp proteins mark injured skeletal muscle in zebrafish. PLoS ONE 7, e31041 (2012).

31 Koibuchi, N. \& Chin, M. T. CHF1/Hey2 plays a pivotal role in left ventricular maturation through suppression of ectopic atrial gene expression. Circ. Res. 100, 850-855 (2007).

32 Koshiba-Takeuchi, K. et al. Reptilian heart development and the molecular basis of cardiac chamber evolution. Nature 461, 95-98 (2009).

$33 \mathrm{Kragl}$, M. et al. Cells keep a memory of their tissue origin during axolotl limb regeneration. Nature 460, 60-65 (2009).

34 Sedmera, D. \& Thompson, R. P. Myocyte proliferation in the developing heart. Dev. Dyn. 240, 1322-1334 (2011).

35 Suster, M. L., Abe, G., Schouw, A. \& Kawakami, K. Transposon-mediated BAC transgenesis in zebrafish. Nat. Protoc. 6, 1998-2021 (2011).

36 Lee, E. C. et al. A highly efficient Escherichia coli-based chromosome engineering system adapted for recombinogenic targeting and subcloning of BAC DNA. Genomics 73, 56-65 (2001).

37 Felker, A. \& Mosimann, C. Contemporary zebrafish transgenesis with Tol2 and application for Cre/lox recombination experiments. Methods Cell Biol. 135, 219-244 (2016)

38 Kwan, K. M. et al. The Tol2kit: a multisite gateway-based construction kit for Tol2 transposon transgenesis constructs. Dev. Dyn. 236, 3088-3099 (2007).

39 Felker, A. et al. In vivo performance and properties of tamoxifen metabolites for CreERT2 control. PLoS ONE 11, e0152989 (2016).

40 Peralta, M. et al. Heartbeat-driven pericardiac fluid forces contribute to epicardium morphogenesis. Curr. Biol. 23, 1726-1735 (2013).

41 Gonzalez-Rosa, J. M. \& Mercader, N. Cryoinjury as a myocardial infarction model for the study of cardiac regeneration in the zebrafish. Nat. Protoc. 7, 782-788 (2012).

42 Mably, J. D., Mohideen, M. A., Burns, C. G., Chen, J. N. \& Fishman, M. C. heart of glass regulates the concentric growth of the heart in zebrafish. Curr. Biol. 13, 2138-2147 (2003)

43 Rohr, S., Otten, C. \& Abdelilah-Seyfried, S. Asymmetric involution of the myocardial field drives heart tube formation in zebrafish. Circ. Res. 102, e12-19 (2008).

44. Mosimann, C. et al. Ubiquitous transgene expression and Cre-based recombination driven by the ubiquitin promoter in zebrafish. Development 138, 169-177 (2012).

45 Gonzalez-Rosa, J. M., Peralta, M. \& Mercader, N. Pan-epicardial lineage tracing reveals that epicardium derived cells give rise to myofibroblasts and perivascular cells during zebrafish heart regeneration. Dev. Biol. 370, 173-186 (2012).

46 Mercader, N., Fischer, S. \& Neumann, C. J. Prdm1 acts downstream of a sequential RA, Wnt and Fgf signaling cascade during zebrafish forelimb induction. Development 133, 2805-2815 (2006).

47 Gonzalez-Rosa, J. M. et al. Use of echocardiography reveals reestablishment of ventricular pumping efficiency and partial ventricular wall motion recovery upon ventricular cryoinjury in the zebrafish. PLoS ONE 9, e115604 (2014).

48 Jahangiri, L. et al. The AP-1 transcription factor component Fosl2 potentiates the rate of myocardial differentiation from the zebrafish second heart field. Development 143, 113-122 (2016).

49 Cai, D., Cohen, K. B., Luo, T., Lichtman, J. W. \& Sanes, J. R. Improved tools for the Brainbow toolbox. Nat. Methods 10, 540-547 (2013).

50 Tessadori, F. et al. Identification and functional characterization of cardiac pacemaker cells in zebrafish. PLoS ONE 7, e47644 (2012).

51 Martin, M. Cutadapt removes adapter sequences from high-throughput sequencing reads. EMBnet.journal 17, (2011). 
52 Li, B. \& Dewey, C. N. RSEM: accurate transcript quantification from RNA-Seq data with or without a reference genome. BMC Bioinformatics 12, 323 (2011).

53 Robinson, M. D., McCarthy, D. J. \& Smyth, G. K. edgeR: a Bioconductor package for differential expression analysis of digital gene expression data. Bioinformatics 26, 139-140 (2010).

\section{Acknowledgements}

We are grateful to the Animal facility, Histology, Microscopy, Cellomics, Bioinformatics, and Genomics Units from CNIC, the Microscopy Imaging Center from the University of Bern; X. Langa, L. Flores, M. Villalba, and R. Costa for experimental assistance; S.

Seyfried and C.-B. Chien for reagents; and M. Torres, S. Martin-Puig, J. González-Sainzde-Aja, R.M. Benedito, and A. Jazwinska for discussion. Funding was through FPU12/ 03007 and BFU2014-56970-P (Plan Estatal de Investigación Científica y Técnica y de Innovación 2013-2016, Programa Estatal de I+D+i Orientada a los Retos de la Sociedad Retos Investigación: Proyectos I+D+i 2016, del Ministerio de Economía competitividad e Industria), and co-funding by Fondo Europeo de Desarrollo Regional (FEDER) (H.S. and N.M.); Swiss National Science Foundation grant 31003A_159721, ANR-SNF collaborative Project 320030E-164245, and the ERC starting grant 337703-zebra-Heart (N.M.). A. E. is enrolled into PhD specialization Cutting Edge Microscopy offered by the Graduate School for Cellular and Biomedical Sciences (GCB) and the Microscopy Imaging Center (MIC). The CNIC is supported by the Ministry of Economy, Industry and Competitiveness (MEIC) and the Pro CNIC Foundation, and is a Severo Ochoa Center of Excellence (MEIC award SEV-2015-0505). Further support was from the Canton of Zürich, project grant from the Swiss Heart Foundation (A.F. and C. Mosimann); the Swiss National Science Foundation (SNSF) professorship (PP00P3_139093) and a Marie Curie Career Integration Grant from the European Commission (CIG PCIG14-GA2013-631984) (C. Mosimann).

\section{Author contributions}

H.S.-I. designed and carried out experiments. M.G.-C., A.S.-M. and A.E. carried out experiments. G.G.-M. carried out ultrasound experiments. A.F. and C. Mosimann generated the drl:mCherry line. C.M. generated the $t b x 5 a: G F P$ and $t b x 5 a: t d T o m a t o$ lines. J.M.G.-R. contributed in the generation of transgenic lines. N.M. designed experiments and secured funding. All authors contributed to writing the manuscript.

\section{Additional information}

Supplementary Information accompanies this paper at https://doi.org/10.1038/s41467 017-02650-6.

Competing interests: The authors declare no competing financial interests.

Reprints and permission information is available online at http://npg.nature.com/ reprintsandpermissions/

Publisher's note: Springer Nature remains neutral with regard to jurisdictional claims in published maps and institutional affiliations.

\begin{abstract}
cc) (i)
Open Access This article is licensed under a Creative Commons Attribution 4.0 International License, which permits use, sharing, adaptation, distribution and reproduction in any medium or format, as long as you give appropriate credit to the original author(s) and the source, provide a link to the Creative Commons license, and indicate if changes were made. The images or other third party material in this article are included in the article's Creative Commons license, unless indicated otherwise in a credit line to the material. If material is not included in the article's Creative Commons license and your intended use is not permitted by statutory regulation or exceeds the permitted use, you will need to obtain permission directly from the copyright holder. To view a copy of this license, visit http://creativecommons.org/ licenses/by/4.0/
\end{abstract}

(C) The Author(s) 2018 